\title{
Similar mechanisms underlie curvature comparison by static and dynamic touch
}

\author{
SYLVIA C. PONT, ASTRID M. L. KAPPERS, and JAN J. KOENDERINK \\ Utrecht University, Utrecht, The Netherlands
}

\begin{abstract}
In four experiments, we tested whether haptic comparison of curvature ranging from $-4 / \mathrm{m}$ to $+4 / \mathrm{m}$ is qualitatively the same for static and for dynamic touch. In Experiments 1 and 3, we tested whether static and dynamic curvature discrimination are based on height differences, attitude (slope) differences, curvature differences, or a combination of these geometrical variables. It was found that both static and dynamic haptic curvature discrimination are based on attitude differences. In Experiments 2 and 4, we tested whether this mechanism leads to errors in the comparison of stimuli with different lengths for static and dynamic touch, respectively. If the judgments are based on attitude differences, subjects will make systematic errors in these comparisons. In both experiments, we found that subjects compared the curvatures of strips of the same length veridically, whereas they made systematic errors if they were required to compare the curvatures of strips of different lengths. Longer stimuli were judged to be more curved than shorter stimuli with the same curvature. We conclude that similar mechanisms underlie static and dynamic haptic curvature comparison. Moreover, additional data comparison showed that static and dynamic curvature comparison is not only qualitatively, but also quantitatively similar.
\end{abstract}

When we touch an object, we get an impression of, for instance, its texture, size, temperature, and shape. The geometrical correlate of the shape of smooth objects is curvature. So, an understanding of curvature perception contributes to the comprehension of the mechanism of shape perception. In this paper, we focus on the manner in which haptic curvature discrimination takes place in the cases of static and dynamic touch. It is evident that the results are interesting in themselves, but, in addition, we are able to compare the two cases in order to investigate whether dynamic curvature comparison follows the same principles as static curvature comparison.

In earlier investigations (Pont, Kappers, \& Koenderink, $1995,1996,1997)$, we tested static haptic discrimination of curved strips for different placements relative to the hand and for different lengths of the stimuli. The strips had a length of 8 or $20 \mathrm{~cm}$, a width of $2 \mathrm{~cm}$, and a curvature ranging from $-1.8 / \mathrm{m}$ to $+1.8 / \mathrm{m}$ (the curvature is the reciprocal of the radius of curvature, and vice versa). Performance in the conditions in which the stimuli were touched with the palmar side of the hand was found to depend pri-

We are grateful to the Netherlands Organization for Scientific Research (NWO) for funding this project. We thank Heino Blanckaert, Jasper Berben, Ronald Burger, Norbert van Woerkom, Michiel Bijlsma, Femius Koenderink, and Inge Lichtenegger for acting as observers and Sheila $\mathrm{McNab}$, whose critical comments have improved the language style of the manuscript. Special thanks go to Hans Kolijn for his technical help. Correspondence concerning this article should be sent to S. C. Pont, Utrecht University, Helmholtz Instituut, Princetonplein 5, 3584 CC Utrecht, The Netherlands (e-mail: a.m.l.kappers@fys.ruu.nl).

-Accepted by previous editor, Myron L. Braunstein marily on contact length, not on the anatomical and neurological structure of the part of the hand with which the stimuli were touched. This trend suggests to us that the effective stimulus for curvature discrimination is the difference in local surface attitude over a curved surfacethat is, the slope difference over the outermost contact positions of the stimulus.

The latter hypothesis led to the experiments that are described in the present paper. If one thinks of possible mechanisms that might underlie curvature perception, there are roughly three possibilities. The first mechanism is based on direct comparison of the curvature (the secondorder geometrical structure); the second is based on comparison of local attitudes (the first-order geometrical structure); and the third is based on comparison of the base-to-peak height difference (the zeroth-order geometrical structure; curvature, local attitude, and base-to-peak height are defined in Figure 5, upper panel). However, it is also possible that curvature perception is based on combinations of these three mechanisms. In Experiment 1, we try to answer whether static curvature discrimination is based on attitude differences exclusively, or whether local curvature and height differences also play a role in curvature perception. In Experiment 3, we study the underlying mechanism for the case of dynamic curvature discrimination.

In the general discussion, the results for static and dynamic touch will be compared, and, in this way, we are able to shed light on the question of whether movements influence performance-in particular, curvature discrimination performance. It is important to note that we do not compare passive and active touch (i.e., being touched and 
to touch, or without and with self-control) but, rather, static and dynamic touch (i.e., without and with movements over the surface), which are both forms of selfcontrolled, or active, touch.

As a first step in haptic curvature discrimination by dynamic touch, the observer puts his or her hand or finger(s) on a surface. Second, the observer moves the hand or finger(s) over the surface. Third, he or she lifts his or her hand off the surface. The main difference between haptic curvature discrimination by static touch and by dynamic touch is that the second step is not taken in the case of static touch. These movements over the surface might provide extra cues for the curvature perception of that surface. For instance, there will be changes in the movement direction of a finger and in the pressure distribution on a finger, if this finger strokes a curved surface. Thus, additional (redundant) transient aspects in the cutaneous and kinesthetic stimulation exist if the hand or finger is moved dynamically over a curved surface, rather than being positioned statically on that surface. It should be noted, however, that self-induced transient aspects of cutaneous and kinesthetic stimulation also exist in the case of static touch-namely, during the phases in which the observer puts his or her hand on and lifts it off the surface.

Several authors, including Gibson (1962), Loomis and Lederman (1986), and Lederman and Klatzky (1987), have reported on differences between passive and active touch. They stress the importance of self-controlled exploratory finger movements. According to Gibson, these movements play a role in isolating invariant relations that specify an object's shape. Lederman and Klatzky suggest that the movements vary with the type of information desired. Loomis and Lederman expect that such movements will lead to a better performance than other manners of touch. On the basis of this literature, we expect haptic curvature discrimination by dynamic touch to be superior to haptic curvature discrimination by static touch.

Only a few psychophysical studies of curvature perception have been done. Here we report the studies that are the most interesting in relation to the present study. Goodwin, John, and Marceglia (1991) investigated tactile discrimination of spherically curved lenticular surfaces that were passively touched with a single fingerpad. The contacted area ranged from a few square millimeters to about $135 \mathrm{~mm}^{2}$. They found that surfaces with curvatures of $+4.9 / \mathrm{m}$ and $-5.4 / \mathrm{m}$ can be discriminated from a flat surface at $75 \%$ correct (a curvature of $5 / \mathrm{m}$ is represented by a circle with a radius of $1 / 5 \mathrm{~m}=.2 \mathrm{~m}$ ). In the present study, we use stimuli with curvatures of between $-4 / \mathrm{m}$ and $+4 / \mathrm{m}$. The curvatures of these stimuli cannot be discriminated statically from flat with a single fingerpad.

Gordon and Morison (1982) investigated discrimination and rating of curved stimuli with a width of $1 \mathrm{~cm}$ and a length of $2-4 \mathrm{~cm}$ that were dynamically touched with the index finger. They found that the threshold was constant if expressed in the base-to-peak height divided by half the length of the stimulus. So, in their experiments, this parameter, which they called the gradient, represented the effective stimulus for curvature. They found a constant gradient of .009 ( $75 \%$ correct). This is equivalent to a constant total attitude difference of about $2^{\circ}$ over the surface. In our experiments on static curvature discrimination, we found that the effective stimulus for curvature is the total attitude difference over the surface (Pont et al., $1996,1997)$. We found that the values for the discrimination thresholds ( $84 \%$ correct), in units of the total attitude difference over the surface, ranged from $1.6^{\circ}$ to $10^{\circ}$, depending on subject and condition.

Davidson (1972) studied active haptic categorization (convex, straight, or concave) of curved strips $20 \mathrm{~cm}$ in length, and Davidson and Whitson (1974) investigated haptic curvature matching for the same strips as those in the previous study. Both studies focused on differences in performance between blind and sighted subjects and related performance to scanning techniques. It was concluded that some scanning techniques lead to greater accuracy than others. Unfortunately, their data do not let us determine exact numerical values for the discrimination thresholds.

Kappers and Koenderink (1996) investigated active haptic discrimination of cylindrically curved hand-sized surfaces. They showed that performance was better than in the former studies, owing to the combined effects of a larger surface area and active, rather than passive, discrimination.

The psychophysical studies described above leave us with two possible outcomes: Performance is better for dynamic touch than for static touch, or performance is the same for the two cases. The literature about possible physiological mechanisms is also important to consider with regard to this topic. As will be clear from the sequel, this literature describes the same two possibilities.

In the literature concerning kinesthesia, the senses of movement and of static position are generally described as different sensation groups (Clark \& Horch, 1986; Ferrell \& Craske, 1992; Gandevia, McCloskey, \& Burke, 1992; Matthews, 1988). There is no general agreement on the question of which receptors or combination of receptors might underlie these senses. Muscle spindles, joint receptors, and cutaneous receptors probably all play a role. The difference between the sense of movement and the sense of static position has been reported on in a more coherent fashion. Gandevia et al. reported that movement enhances kinesthetic acuity, and Clark and Horch stated that the awareness of movement has a more vivid character than the sense of position. In comparison with static touch, dynamic touch might lead to a more accurate perception of the (changes in the) posture of the hand on the curved surface and, possibly, of the curvature of that surface. It might also lead to a more accurate perception of the length over which a stimulus is touched. This might result in a better performance in comparisons of the curvatures of surfaces of different lengths. On the basis of this literature, we expect that performance for curvature comparison will be better for dynamic than for static touch.

Johnson, Hsiao, and Twombly (1996) reported that tactile form recognition is little affected by the manner of 
contact between the skin and the stimulus. LaMotte, Srinivasan, $\mathrm{Lu}$, and Klusch-Petersen (1994) found that cutaneous neural codes for shapes that were stroked across the fingerpads of monkeys are probably invariant with moderate changes in the way the object contacts the skin. Thus, these authors suggest that static curvature discrimination might not differ from dynamic curvature discrimination, because the cutaneous sensitivity is similar.

The psychophysical and physiological studies that we considered leave open two possibilities: Curvature comparison performance is better for dynamic than for static touch, or the performance is similar for the two cases.

The aims of the present investigation were fivefold and eventually resulted in a comparison of the mechanisms that underlie static and dynamic touch. First, we wanted to test whether curvature discrimination depends on local surface attitude differences exclusively, or whether local curvature or base-to-peak height differences also play a role in this process. In other words, is curvature discriminated on the basis of the first-order geometrical properties of the stimulus only, or is there also an influence of the second- and zeroth-order geometrical structure? We sought answers to these questions by measuring and comparing the discrimination thresholds for three series of stimuli with different geometrical properties: one series in which only the zeroth-order structure (base-to-peak height difference) varied, one in which the geometrical properties up to the first-order structure (slope or attitude) varied, and one in which the geometrical properties up to the second-order structure (curvature) varied (Experiment 1 ). Thus, the three geometrically different categories of stimuli were constructed so that the geometrical second-and first-order components were left out systematically, until only the zeroth-order component remained. Consequently, we are able to answer the question of whether these geometrical components contribute to the discrimination of curvature. If the discrimination thresholds increase if a geometrical component of the stimulus is removed, it must be concluded that this component contributes to the performance.

Second, we studied the manner in which stimulations of the different fingers are combined. We did this by measuring the curvature discrimination thresholds under conditions in which the number of fingers contacting the stimuli was varied systematically between one and three (Experiment 1).

The third aim was to investigate whether the subject was aware of the positions of the fingers on the stimuli and took them into account. We therefore measured points of subjective equality for curved strips that were touched over different lengths (Experiment 2). We tested conditions in which the reference strips were touched with the fingers held close together and the test strips with the fingers spread out wide, and vice versa.

Fourth, we wanted to investigate whether dynamic curvature discrimination depends on the length of the stimulus. We therefore tested the discrimination of curved strips from a flat strip for four lengths of strips: $5,10,15$, and $20 \mathrm{~cm}$ (Experiment 3 ). In addition, we analyzed whether it was possible to indicate the effective stimulus for the discrimination of curvature under these conditions (as we do in Experiment 1 for the case of static touch). If curvature discrimination is enacted on the basis of curvature (attitude differences, height differences), the thresholds expressed in terms of curvature (attitude differences, height differences) will be constant. We discuss whether the data from Experiment 3 can be described by one of these limit cases. We therefore calculated the curvature discrimination thresholds in terms of height difference, attitude difference, and radius of curvature.

Fifth, we studied whether subjects were able to match the curvatures of strips of different lengths by dynamic touch. In Experiment 4, we tested subjects' matching of the curvatures of strips of the same length and of different lengths. The subjects had to match the curvature of a strip, ranging from $-1.6 / \mathrm{m}$ to $+1.6 / \mathrm{m}$, with another strip. All nine possible combinations of the strip lengths of 5 , 10 , or $20 \mathrm{~cm}$ were tested. We tried to find out whether the subjects took a difference in the length of strips into account in the matching of their curvatures. We therefore compared the results of the matching experiment to predictions made on the basis of models.

The comparison of the results of Experiments 1 and 2 and those of Experiments 3 and 4 will shed light on the question of whether subjects take the stimulus length into account in the cases of static and dynamic curvature comparison. By comparing the results of Experiments 1 and 2 with those of Experiments 3 and 4, respectively, we are able to study whether the same, or at least similar, mechanisms underlie static and dynamic curvature discrimination. In the general discussion, we will compare static and dynamic curvature comparison in a qualitative (with regard to the underlying mechanisms) and quantitave (with regard to the values of the discrimination thresholds) manner.

\section{EXPERIMENT 1}

The aim of Experiment 1 was to answer the question of whether static discrimination of curved strips from flat ones is only based on local surface attitudes or whether local curvature or base-to-peak height difference also play a role in the discrimination. Furthermore, we studied the manner in which the stimulations of the different fingers are combined. We therefore tested discrimination of stimuli with geometrical components up to the second, first, or zeroth order under conditions in which the combination of fingers contacting the stimuli was varied.

Figure 1 shows schematically what kind of stimuli we used and what their geometrical properties were. The curvature, attitudes, and height differences in Figure 1 are much larger than those of the real stimuli. Note that a discrimination threshold that is measured using a certain kind of stimulus can be represented in geometrical components that correspond to the geometrical components of that stimulus. So, a discrimination threshold that 


\section{stimulus:}

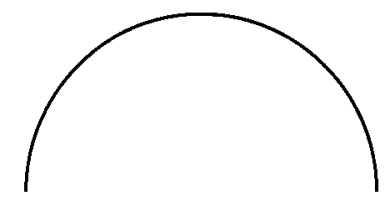

second-order

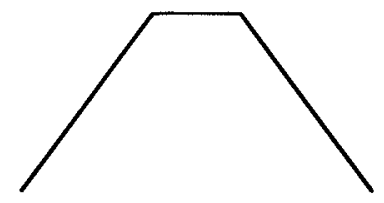

first-order

\author{
geometrical components of \\ stimulus / possible determinants \\ of thresholds:
}

\author{
- base-to-peak height difference \\ - attitude difference \\ - curvature
}

- base-to-peak height difference

- attitude difference

attitude difference

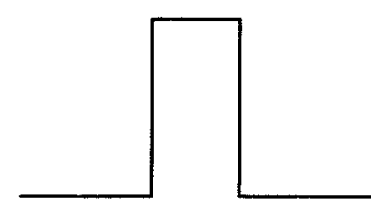

- base-to-peak height difference

zeroth-order

\begin{abstract}
Figure 1. Schematic representation of three geometrically different categories of stimuli. For clarity, the strips are depicted with much larger curvatures, attitudes, and height differences than they have in reality. The geometrical properties of the stimuli and, thus, of the possible determinants of the thresholds that were measured using these stimuli are described at the right side of the second-order strips (curved stimuli), the first-order strips (trapezoidal stimuli), and the zeroth-order strips (rectangular stimuli).
\end{abstract}

is measured using curved (second-order) stimuli can be represented in terms of curvature, local attitudes, and base-to-peak height difference (the local attitudes under and the base-to-peak height difference between fingers that touch a curved stimulus can be calculated exactly, if the positions of the fingers on a curved stimulus are known; see Figure 5). A threshold that is measured using first-order stimuli can be represented in terms of base-topeak height difference (this can be calculated exactly), but not in terms of curvature (a transformation to curvature would need an assumption about the manner in which a curve fits these stimuli and would thus be arbitrary). A threshold that is measured using zeroth-order stimuli can only be represented in terms of base-to-peak height difference (transformations to curvature or local attitudes would be arbitrary). The discrimination thresholds can only be compared if they are represented in identical units.

If curvature discrimination is based on the second-order geometry (curvature), the thresholds for second-order stimuli will be lower than those for the first- and zerothorder stimuli (if compared in appropriate units). If curvature discrimination is based on the first-order geometry, the thresholds (in units of local attitude) for the first- and second-order stimuli will be the same, but those (in units of base-to-peak height difference) for the second-order stimuli will be lower than those for the zeroth-order stimuli. If curvature discrimination is based on the zerothorder geometry, the thresholds (in units of base-to-peak height difference) for zeroth-order stimuli will be the same as those for first- and second-order stimuli. If curvature discrimination is based on a combination of geometrical components, the results will be somewhere in between these three extremes. As a final verification for the possibility that the second-order geometry contributes to the curvature discrimination, we tested the discrimination of local curvature in a control condition. This possibility must be excluded if the discrimination thresholds for this control condition indicate that it is impossible to discriminate the curvatures locally in our measuring range. 

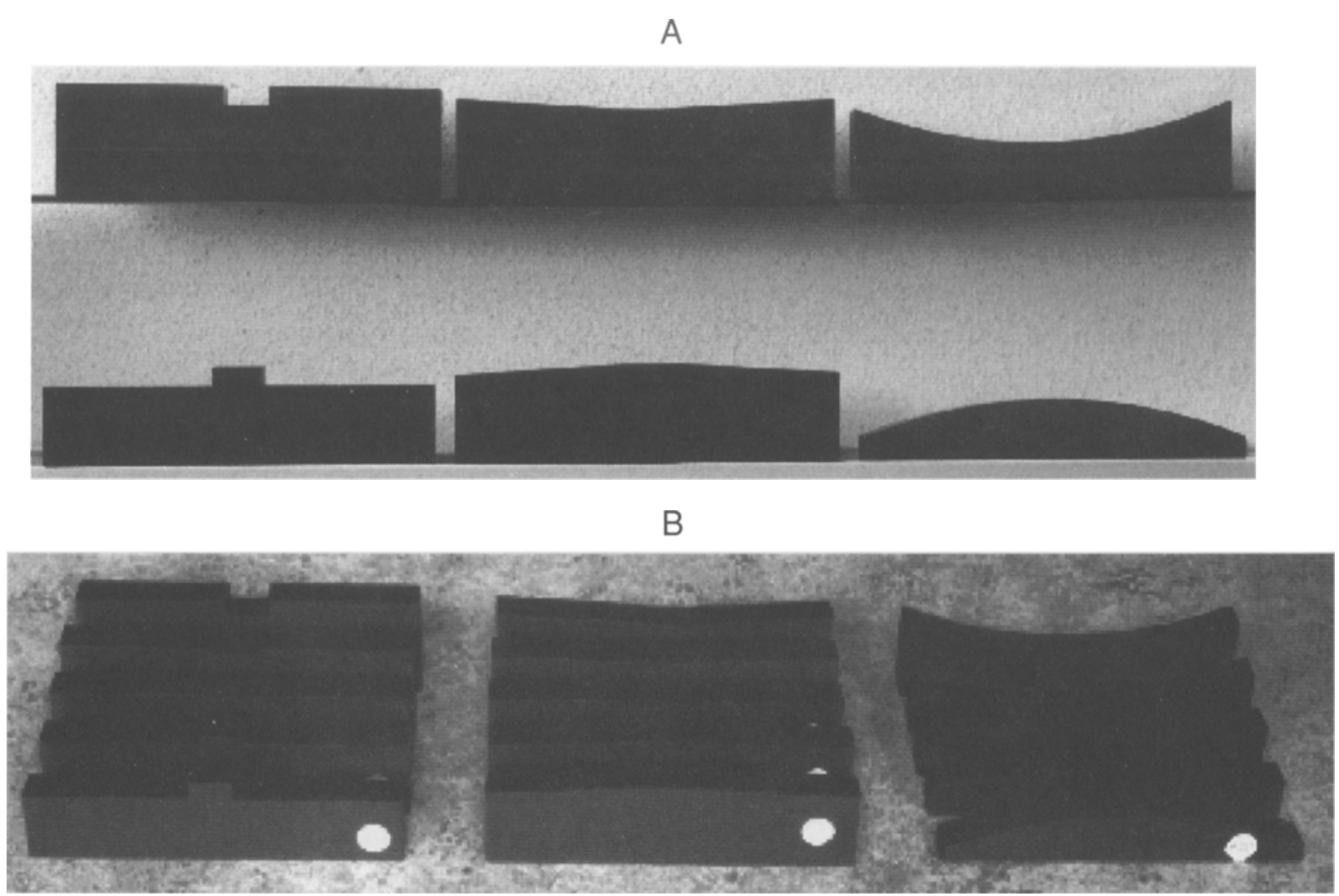

Figure 2. The three sets of stimuli with different geometrical properties. The zeroth-order stimuli are depicted on the left side, the first-order stimuli in the middle, and the second-order stimuli on the right side. The most concave versions of the stimuli are the upper strips in Panel $A$ and those at the rear of Panel B. The most convex ones are the lower strips in Panel $A$ and those at the front of Panel $B$.

On the basis of earlier experimental results, we expected to find that curvature discrimination is based on local attitudes (the first-order geometry).

\section{Method}

Subjects. Four naive subjects participated in the experiments. They were paid for their efforts. All the subjects were unaware of the objectives of the experiment. Subjects R.B., J.B., and N.W. are right-handed; subject H.B. is left-handed (according to definitions used by Coren, 1993, who devised a standard questionnaire). Subjects R.B., J.B., and H.B. were familiar with psychophysical measurements and with the experimental task because they had participated in pilot experiments for about $3 \mathrm{~h}$. Subject N.W. had acted as an observer in previous haptic curvature discrimination experiments but was naive in all other respects.

Stimuli. The haptic stimuli were PVC strips with a length of $20 \mathrm{~cm}$, a width of $2 \mathrm{~cm}$, and a peak or trough height of about $5 \mathrm{~cm}$. The peak or trough is located in the middle of the strip, and, within a stimulus set, the middle of each strip is always at exactly the same height. Three sets of stimuli with different geometrical properties were used. The strips are depicted in Figure 2. One set consisted of strips with a $2.5-\mathrm{cm}$-wide raised or sunken part in the middle of the strip, ranging from $-10 \mathrm{~mm}$ to $+10 \mathrm{~mm}$. These rectangular stimuli will be called zeroth-order stimuli (the stimuli on the left in Figure 2), because they are parameterized by the zeroth-order geometrical structure. In a second series of strips, each strip had an attitude ranging from $-3.4^{\circ}$ to $+3.4^{\circ}$, relative to the horizontal, on the left and the right side of the strip, so that each strip was symmetrical. These stimuli also had a $2.5-\mathrm{cm}$-wide horizontal platform in the middle. The first-order geometrical structure parameterizes these trapezoidal shapes, which will therefore be called first-order stimuli (the middle strips in Figure 2). The third set consisted of circularly curved strips with a curvature ranging from $-4 / m$ to $+4 / m$ (the curvature, the second-order parameter, of a circularly curved strip is constant over its length and, therefore, equal to the local curvature). These strips are parameterized by the second-order geometrical structure and will therefore be called second-order stimuli (the stimuli on the right in Figure 2).

Note that the second-order stimuli have first- and zeroth-order components that covary with the second-order parameter (strips with larger curvatures have larger local attitudes and a larger baseto-peak height difference). Also, the first-order stimuli have a zeroth-order component that covaries with the first-order parameter (the base-to-peak height difference is larger in the case of trapezoidal shapes with larger attitudes).

The most concave versions of the stimuli are the upper strips in Figure 2A and those at the rear of Figure 2B. The lower strips in Figure 2A and those in the front of Figure $2 \mathrm{~B}$ are the most convex ones. The strips in the middles of each of the three series of stimuli in Figure 2B are flat. Thus, this panel shows the complete range for each of the stimuli series but not the complete series (each series contains at least 41 strips; the stepwidth between stimuli is thus much smaller than that shown in Figure 2B).

Experimental setup and procedure. The subjects were seated behind a curtain that prevented them from seeing the experimenter 

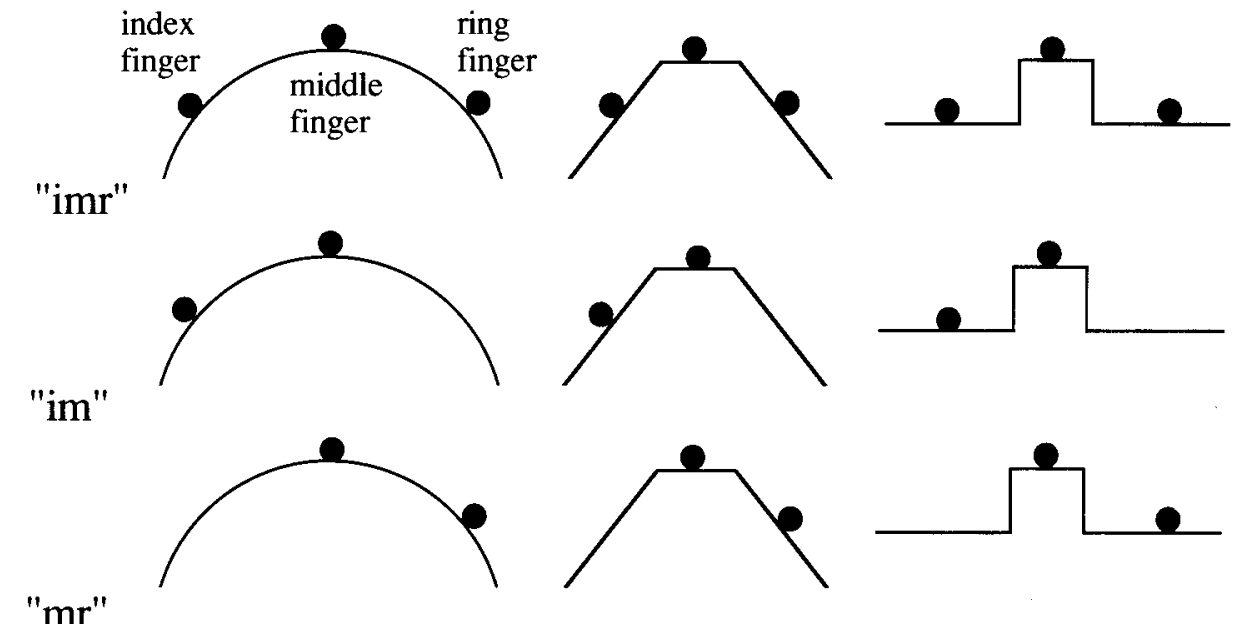

"mr"
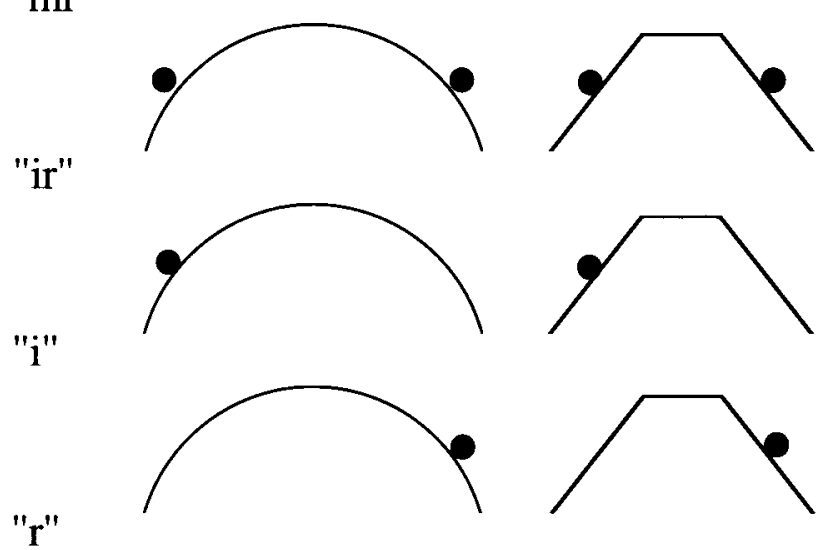

\section{control conditions:}
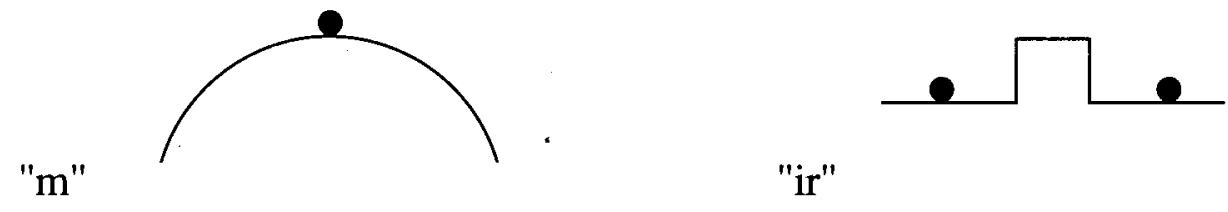

Figure 3. Schematic representation of the conditions and control conditions that were tested in the first experiment. For clarity, the strips are depicted with much larger curvatures, attitudes, and height differences than they have in reality. Only convex stimuli are shown. The filled circles represent the index (i), middle ( $m$ ), and ring ( $r)$ fingers.

and the stimuli. We chose to use a curtain, rather than to blindfold the subjects, because they were more comfortable with this setup during the long experiments. Moreover, this setup enabled the subjects to write down their judgments themselves. Consequently, the experimenter needed less time per trial. As a result, it was possible to present 112 trials in about $15 \mathrm{~min}$. This was a comfortable speed for the subjects, who were allowed to (statically) touch the stimuli for as long as they liked. However, they took only a little time (about $8 \mathrm{sec}$ per trial) to make their judgments.

The subjects put their hands under the curtain to touch the stimuli presented by the experimenter. They rested their elbows on the table and moved their relaxed hands up from and down onto the stimuli. The strips were touched in a static way, meaning that the subjects put their hands on, but did not move their hands over, the stimuli. The strips were fixed in a frame so that they were always presented in the same place: at right angles to the subject's fingers, under the distal joints of the index, middle, and ring fingers. The middle finger was always positioned at the middle (the peak or the dale) of the strips. This was enforced by means of two small laths (thin narrow strips), which were fixed vertically above the place at which the strips were to be touched and between which the middle finger could be moved up from and down onto the stimuli. The positions of the stimuli and of the fingers on the stimuli were checked by the experimenter. 
The task of the subjects was to touch two successively presented stimuli and to indicate which of the two strips was the more convex. They did this by writing down " 1 " or " 2 " on a response sheet. To familiarize the subjects with the concepts convex and concave, we visually showed them examples of these stimuli before each test. We ascertained that the subjects did not have difficulties with these concepts. Before each test, they also did some trials on combinations of stimuli that were at extremes of the measuring ranges. Feedback was given on these trials, and a session was started after they had correctly judged four of these combinations successively. We did not give feedback during the actual experiments.

Discrimination performance for the second-and first-order stimuli was tested under six finger conditions and for the zeroth-order stimuli under three finger conditions. The finger conditions are depicted schematically in Figure 3. For clarity, the strips are depicted with much larger curvatures, local attitudes, and base-to-peak height differences than they have in reality, and only for convex cases. In finger condition "imr," the stimuli were touched with three fingersthe index, middle, and ring fingers. In the finger conditions "im" and " $m r$," the strips were touched with the middle finger and either the index or the ring finger. The finger conditions in which the stimuli were touched with the index and ring fingers ("ir"), the index finger only ("i"), or the ring finger only (" $r$ ") were tested only for the first- and second-order stimuli. These finger conditions were not tested for the zeroth-order stimuli, because the only remaining cue under these conditions would have been the height difference between successively presented strips. In a control condition, we tested whether the subjects could discriminate this cue (the height difference between successively presented strips). In this condition, the zerothorder stimuli were touched with the index and ring fingers ("ir").

In another control condition, we tested whether the subjects could discriminate curvature locally, in the range of $-4 / \mathrm{m}$ to $+4 / \mathrm{m}$. In this condition, the second-order stimuli were touched with the middle finger only ("m"). Local curvature might, of course, also be a cue in all the other finger conditions in which the second-order stimuli are used, but in those cases, the local attitudes also give information about the curvature. Comparison of the results for these finger conditions with the corresponding ones for the first-order stimuli will tell us whether the extra cue of local curvature leads to improved performance in the case of the second-order stimuli. However, the local curvature is the only remaining cue in the control condition " $m$," and thus, it tests whether local curvature can be discriminated at all in our measuring range. The base-to-peak height difference provides another possible cue in finger conditions "imr," "im," and "mr," in the case of both the second- and the first-order stimuli. Comparison of these results with the corresponding values for the zeroth-order strips will then tell us whether the base-to-peak height difference plays a role in the discrimination of these stimuli.

In each condition, a flat reference strip was combined with 14 test strips, 7 convex and 7 concave stimuli. Because the discrimination thresholds differed for the different conditions and subjects, the measuring range was adjusted after each test, on the basis of the performance in the previous test. All the combinations were presented eight times: four times in one order (first the reference and second the test strip) and four times in the other order (first the test and second the reference strip) in a random sequence. Each experimental condition thus consisted of 14 (test strips) $* 8$ (presentations) $=112$ trials and was tested three times on different days except for the control conditions, which were tested once. Subjects H.B., R.B., and J.B. participated in all these experiments. For subject N.W., we only tested condition "imr" (once). The different conditions were tested in a random order. With subjects H.B. and R.B., we tested discrimination performance for the second-order stimuli first and after that for the zeroth- and first-order stimuli (mixed). With J.B., we tested all the conditions in a random order. This experiment consisted of about $40 \mathrm{~h}$ of measurements.
Analysis. Cumulative Gauss distributions were fitted as psychometric curves to the percentages of judgments in which the test strips were judged to be more convex than the reference strip as a function of the curvature (or attitude or height difference) of the test strips, relative to the reference strip. The Levenberg-Marquardt method (Press, Flannery, Teukolsky, \& Vetterling, 1988) was used to fit the data. Figure 4 shows a representative example of how the curves fit the data. A psychometric curve can be characterized by its location $(\mu)$ and its slope $(\sigma)$. The location $(\mu)$, or the point of subjective equality, represents the $50 \%$ point, or the mean (Macmillan \& Creelman, 1991). The value of $\sigma$ is inversely related to the steepness of the curve and represents the discrimination threshold at $84 \%$ correct (Macmillan \& Creelman, 1991). We calculated the means and the standard errors over the parameters of the separate curves for each of the three measurements per condition.

The curves were determined for percentages of judgments as a function of the curvature, the attitude, or the base-to-peak height difference of the stimuli (the variable that is on the horizontal axis; see Figure 4). Thus, the curve parameters for the data on the second-, first-, and zeroth-order stimuli were represented in units of curvature, attitude, and base-to-peak height difference, respectively. These values could not be compared directly but had to be represented in a uniform framework.

Transformations and measurement of contact positions. In order to be able to compare the thresholds for the geometrically different stimuli, we transformed the values for the second-order stimuli (in terms of curvature) into values in terms of the first- and zeroth-order structures (local attitude and base-to-peak height difference), using the spacing between the fingers. The definition of the first- and zeroth-order structures in the case of a second-order stimulus is depicted in the upper panel of Figure 5. This figure shows a schematic representation of three fingers touching a curved strip (the three fingers and convexness are arbitrary choices). The

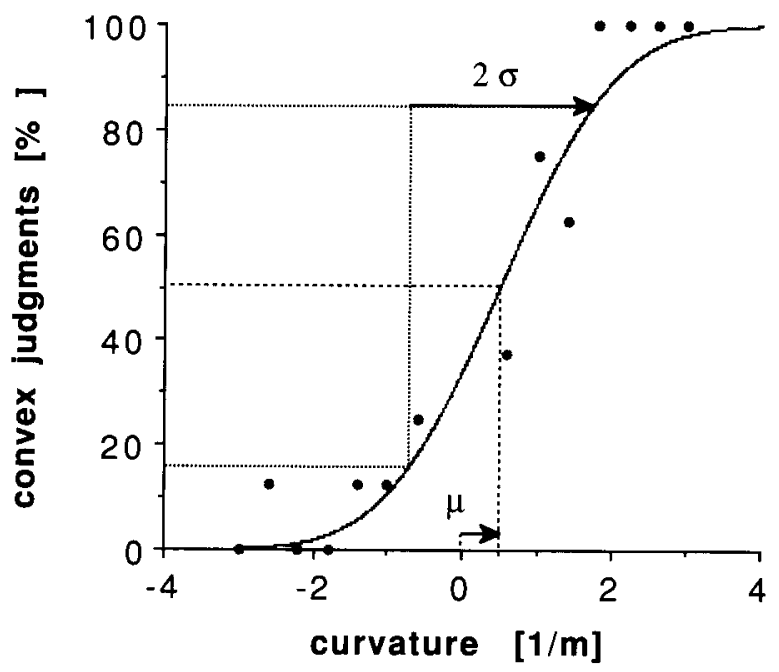

Figure 4. A representative example of datapoints and the psychometric function that was fitted to these datapoints. On the vertical axis are the percentages of judgments in which the test shapes were judged as more convex than the reference shape. On the horizontal axis are the curvatures (or attitudes, or base-topeak height differences) of the test strips, relative to the reference strip. $\sigma$, or the discrimination threshold at $84 \%$ correct, is the difference between the $84 \%$ and the $50 \%$ points, which is inversely related to the steepness of the curve. $\mu$ is the $50 \%$ point, or the point of subjective equality. 

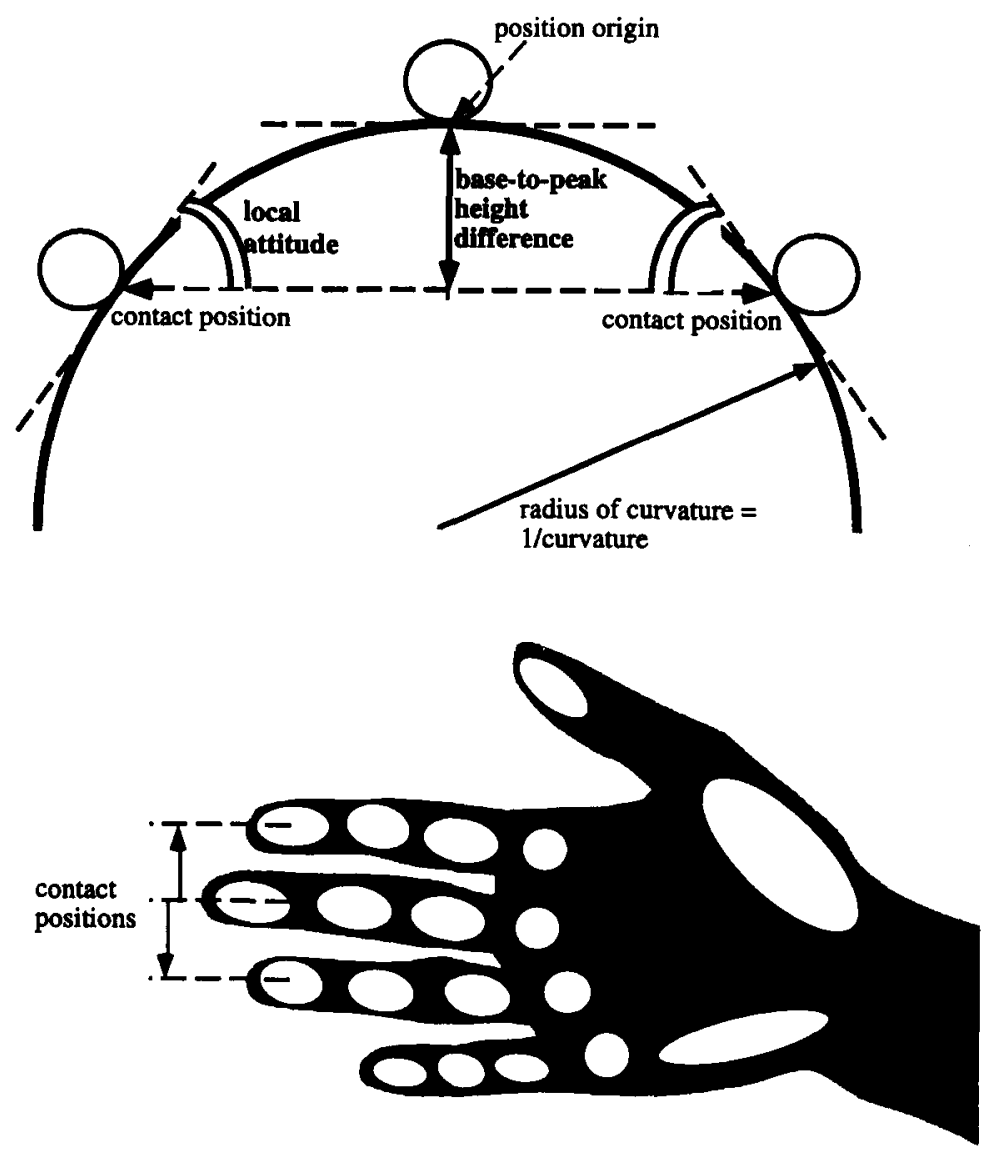

Figure 5. The definition of the first- and zeroth-order structures in the case of a second-order strip and the definition of the contact positions. The upper panel shows a schematic representation of three fingers touching a convex curved stimulus (the three fingers and convexness are arbitrary choices). The zeroth-order component is the base-to-peak height difference over the touched part of the strip. The first-order components are the local attitudes at the positions on the fingers where they contact the stimuli. The lower panel shows a photocopy of a hand, with a schematic representation of the contact areas of the hand with the surface of the photocopier showing up white. The contact positions are defined as the centers of these contact areas.

zeroth-order component is the base-to-peak height difference over the touched part of the strip. In other words, it is the height difference between the position where the middle finger and the positions where the index and ring fingers contact the stimulus (just as in the case of the zeroth-order stimuli). The first-order components are the local attitudes at the positions of the index and ring fingers, relative to the horizontal (analogous to the case of the first-order stimuli). It will be clear from this figure that these variables can be calculated if the curvature (or its reciprocal, the radius of curvature) and contact positions are known.

The contact positions of the fingers on the strips were determined in the following manner. We made a real-size photocopy of each subject's hand. The contact areas of the hand with the surface of the photocopier show up white in these images. As is shown in Figure 5, lower panel, we measured contact positions as the centers of these areas, relative to the position of the middle finger (that always contacted the middle of the stimuli, which was the position of the origin in our calculations). Sometimes the distances between the middle and ring fingers and the middle and index fingers differed. Then, the local attitudes and base-to-peak height differences on the posi- tions of the index and ring fingers differed too. In those cases, we calculated the means of the two values for the local attitude and of the two values for the base-to-peak height difference. We checked the measurements of the contact lengths by estimating the positions of the fingers while the subjects touched the strips in the experimental setup. Finally, we calculated standard errors in terms of local attitude and base-to-peak height difference for all transformed datapoints.

\section{Results}

The means, or points of subjective equality, for the first experiment do not differ systematically from the reference values. This was expected, because possible influences of biases were counterbalanced in this experiment.

The discrimination thresholds are represented in Figure 6. The results for R.B., J.B., and H.B. are shown in the left, middle, and right panels, respectively. In the upper panels, the values for the first-order stimuli are shown together with those for the second-order stimuli, both ex- 

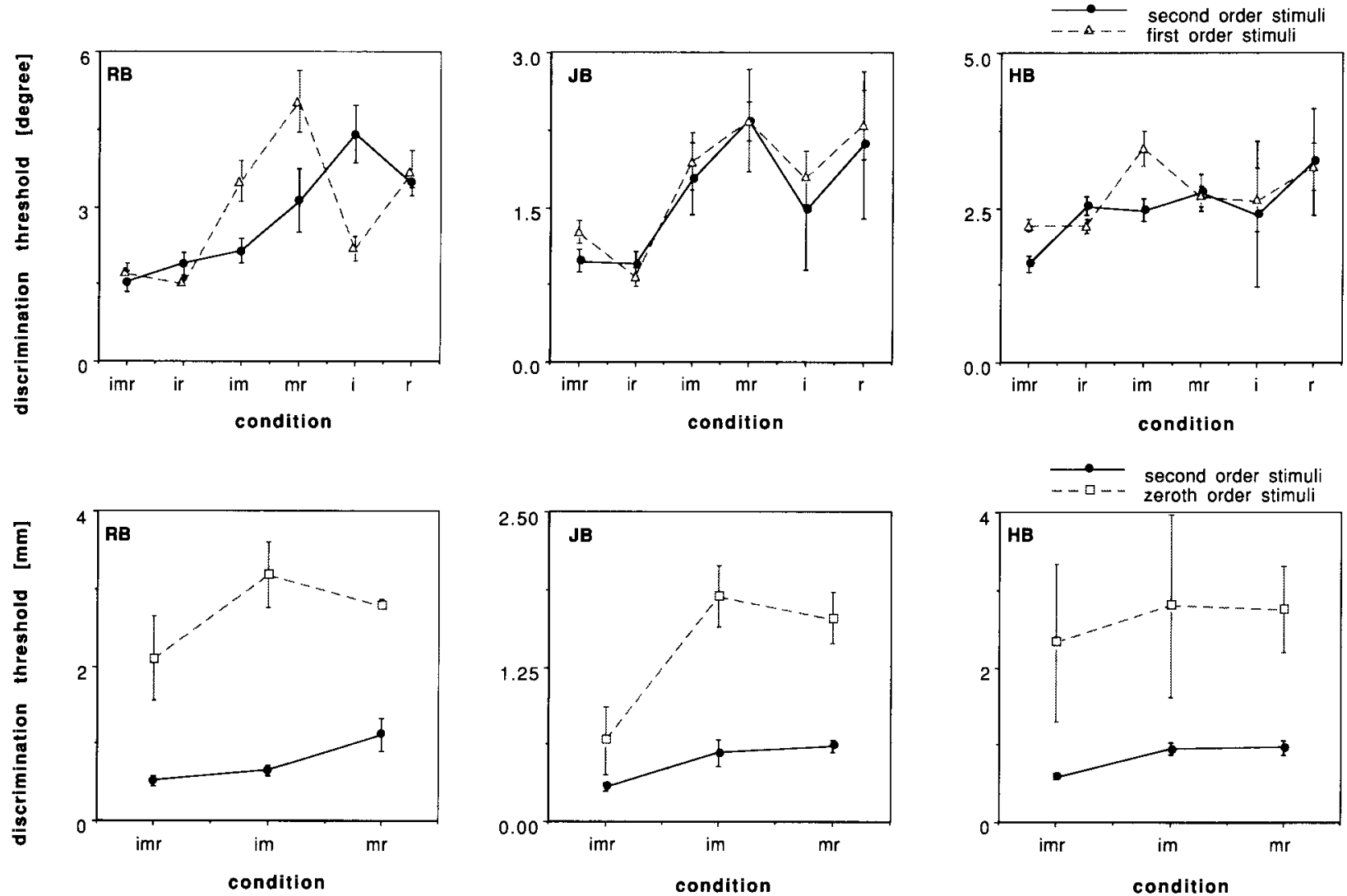

Figure 6. The discrimination thresholds for the conditions that were tested in Experiment 1. The connections between the thresholds for the different conditions are for visualization of the data only. The results for R.B., J.B., and H.B. are shown in the left, middle, and right panels, respectively. In the upper panels, the values for the first-order stimuli are shown together with those for the second-order stimuli in terms of local attitude. The results for the zeroth-order stimuli and those for the second-order stimuli in terms of base-to-peak height difference are depicted in the lower panels. The letters that correspond to the conditions in Figure 3 are on the horizontal axis.

pressed in terms of local attitude. It is clear that the thresholds for the second-order stimuli in terms of local attitude closely resemble those for the first-order stimuli. This is particularly clear for J.B. and H.B., and somewhat less so for R.B. An inspection of the upper panels of Figure 6 shows that the thresholds for the discrimination of the curved stimuli, having properties up to the second-order geometrical structure, are not systematically lower than those for the first-order stimuli, having properties up to the first-order geometrical structure. To investigate whether the geometrical structure (order) of the stimuli, the finger combination, and the subject had significant effects on the discrimination thresholds in terms of local attitude, we performed a two-way related analysis of variance (ANOVA). The geometrical structure of the stimuli (first- or secondorder) does not have a significant effect on the thresholds, in terms of local attitude $[F(1,10)=0.76, p>.25]$.

The results for the zeroth-order stimuli and for the second-order stimuli in terms of base-to-peak height difference are depicted in the lower panels of Figure 6 . The thresholds for the zeroth-order stimuli are clearly much higher than those for the second-order stimuli, in terms of base-to-peak height differences. To test whether this and other effects on the discrimination thresholds in terms of height difference were significant, we performed another two-way related ANOVA. The geometrical structure of the stimuli (zeroth- or second-order) has indeed a significant effect on the thresholds expressed in terms of baseto-peak height difference $[F(1,4)=259.37, p<.001]$. Thus, if the subjects are able to discriminate pure height differences as in the case of the zeroth-order stimuli, these differences have to be much larger than the zeroth-order component of a curved stimulus at threshold level. We conclude that base-to-peak height difference cannot be a cue for curvature discrimination in our measuring range (at most, $-4 / \mathrm{m}$ to $+4 / \mathrm{m}$ ).

In all cases, the effects of the finger combination and of the subject were significant [thresholds in terms of local attitudes: finger combination, $F(5,10)=6.46, p<.01$; subject, $F(2,10)=11.97, p<.01$; thresholds in terms of baseto-peak height difference: finger combination, $F(2,4)=$ $16.19, p<.025$; subject, $F(2,4)=30.93, p<.01]$. The differences between the subjects are clear from Figure 6; note, for instance, the difference in the threshold ranges for subject J.B. and, on the other hand, those for R.B. and H.B. The effects of the finger combination are due to the overall increase in the threshold values for cases in which fewer fingers are used (Figure 6, from left to right in each 


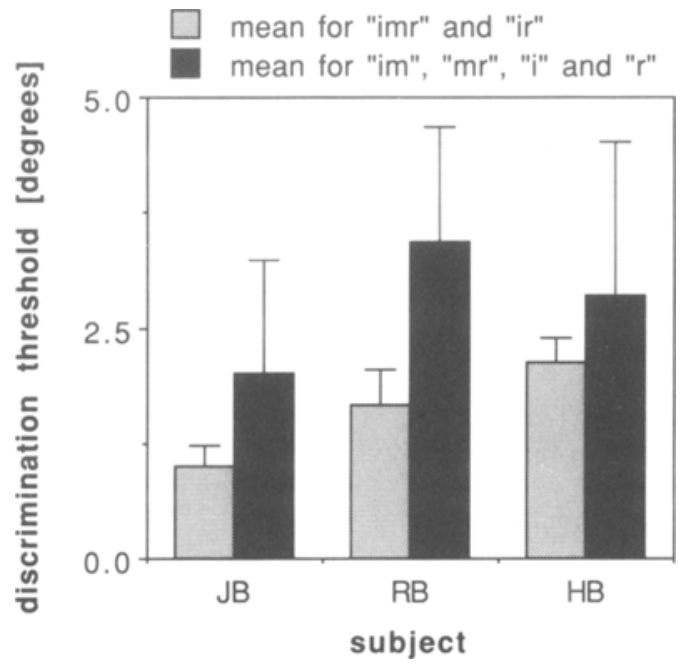

Figure 7. The averages for both the first- and second-order strips of the tl "esholds for conditions "imr" and "ir" (black bars) and for "im," "mr," "i," and "r" (gray bars), in degrees. On the horizontal axis are the 3 subjects who participated in Experiment 1.

panel). There was one significant interaction in the case of the zeroth-order terms [geometrical structure of the stimulus $\times$ subject, $F(2,4)=11.72, p<.025]$.

Closer examination of the thresholds expressed in terms of local attitude for the different conditions (Figure 6, upper panels) reveals some further results. First, the thresholds for the different conditions differ at most by a factor of 3.3. Second, the overall performance for the conditions " $i$ " and " $r$ " does not differ systematically from that for "im" and "mr." Thus, the local attitudes can be determined by means of only one finger, and additional stimulation of the middle finger (under which the local attitude is horizontal) does not improve this judgment. The fact that subjects can discriminate the stimuli at all under the conditions " $i$ " and " $r$ " proves that they must use foreknowledge about the positions of their fingers relative to the middle of the strips. Third, the results for "imr" and "ir" do not differ systematically. So again, stimulation of the middle finger does not improve performance. In Figure 7, we compare the averages for both the first- and the second-order strips of the thresholds for conditions "imr" and "ir" with those for "im," "mr," "i," and "r." The mean thresholds for the conditions in which both the index and the ring finger touched the stimuli (conditions "imr" and "ir") are lower than the values for the cases in which only one of these fingers was stimulated. Thus, performance improves if the stimulation consists of two nonhorizontal attitudes instead of one. The difference between the two means is about a factor of two for J.B. and R.B., and somewhat less for H.B.

The thresholds for control condition "ir" for the zerothorder stimuli were 6.6 (R.B.), 2.7 (J.B.), and $3.6 \mathrm{~mm}$ (H.B.). These values are higher than the thresholds for all the other conditions tested for these stimuli and these subjects. So, discrimination of height differences be- tween successively presented strips is poorer than that of instantaneous height differences between fingers (the base-to-peak height differences). It is, therefore, the instantaneous height difference between the fingers that primarily determines the thresholds for the zeroth-order stimuli.

The control condition " $m$ " was tested for the secondorder stimuli. This resulted in thresholds of $10.5 / \mathrm{m}$ (R.B.), $8.3 / \mathrm{m}$ (J.B.) and $14.5 / \mathrm{m}$ (H.B.). These values exceed the measuring range (the largest absolute curvature we used was $4 / \mathrm{m}$ ) and, thus, are not well defined. However, it is clear from these results that, in our measuring range, the subjects cannot determine curvature locally.

\section{Discussion}

One can think of several ways in which curvature discrimination takes place. Here, we discuss several of these possibilities and try to find out whether these mechanisms are involved in our experiments.

A relatively simple mechanism for discriminating curvature might be based on the local curvature. Our results show, however, that this cannot be the case in our experiments, because the subjects could not discriminate the local curvature for the second-order stimuli in our measuring range $(-4 / \mathrm{m}$ to $+4 / \mathrm{m})$. This agrees with the results of Goodwin et al. (1991), who showed that the thresholds ( $75 \%$ correct) for the discrimination of a flat surface from curved surfaces that were pushed onto the fingerpad of the index finger were $+4.9 / \mathrm{m}$ and $-5.4 / \mathrm{m}$. Thus, the curvature discrimination cannot take place via a mechanism that is based on local curvature.

Another relatively simple mechanism is curvature discrimination on the basis of base-to-peak height differences. The discrimination thresholds for the zeroth-order stimuli were of the order of 2-3 mm. For the second-order stimuli, the discrimination thresholds expressed in terms of base-to-peak height differences are from 0.5 to $1 \mathrm{~mm}$. This difference in threshold values indicates clearly that the additional geometrical structure in the case of the second-order strips is used for improving performance. Thus, in our experiment, the second-order stimuli cannot be discriminated on the basis of base-to-peak height differences alone. The thresholds in units of base-to-peak height differences can be transformed into a rotation about a metacarpophalangeal joint. In the case of a straight finger with a length of about $9 \mathrm{~cm}$, this rotation is of the order of $1^{\circ}-2^{\circ}$ for the zeroth-order stimuli, or $0.3^{\circ}-0.6^{\circ}$ for the second-order stimuli. It is difficult to compare these values with those measured in investigations into the kinesthetic sense, because those values depend heavily on the experimental conditions and task. But, if subjects have to identify the direction of the movement of the middle finger, the thresholds are in the range of $1^{\circ}-6^{\circ}$ (Clark \& Horch, 1986). The joint rotations in the case of the secondorder strips would, thus, be too small to identify the movement direction. This is in agreement with our results. Thus, in our experiment, the curvature cannot be discriminated via a mechanism that is based on base-to-peak height differences alone. 
The conclusions concerning the roles of base-to-peak height differences and local curvature depend on the range of curvatures used in the experiment. Curvatures smaller than $-5.4 / \mathrm{m}$ or larger than $+4.9 / \mathrm{m}$ exceed the thresholds at $75 \%$ correct (or $-7.6 / \mathrm{m}$ and $+6.9 / \mathrm{m}$ at $84 \%$ correct) for discriminating local curvature from flatness (Goodwin et al., 1991). The height differences over the touched parts of curved stimuli exceed the thresholds for discrimination of base-to-peak height differences from curvatures of about $3 / \mathrm{m}-4 / \mathrm{m}$. We expect that the local curvature or baseto-peak height differences can be used as extra cues to discriminate curvatures from about $7 / \mathrm{m}$ or $3 / \mathrm{m}$, respectively. In those cases, curvature discrimination performance might improve for larger curvatures.

A third way in which the curvature discrimination might take place involves time differences between the moments at which the different fingers contact a stimulus. When a convex stimulus is touched, the middle finger might contact the strip before the index and ring fingers, and afterwards in the case of a concave strip. However, we find that it is possible to discriminate the strips by means of only one finger, and, in conditions "i" and " $r$," such time differences do not exist. Furthermore, performance for the zeroth-order stimuli is poorer than for the second- and first-order stimuli (Figure 6, lower panels), whereas the time difference should be larger for the zerothorder strips because of the larger base-to-peak height differences. Thus, in our experiment, the curvature is not discriminated via a mechanism that involves the time differences between the moments at which the fingers contact the stimuli.

Our results strongly indicate that curvature discrimination in the range $-4 / \mathrm{m}$ to $+4 / \mathrm{m}$ must take place via a mechanism that is based on local attitudes. The thresholds for the second- and first-order stimuli resemble each other closely. The average values were $2.8^{\circ}$ or $1.6^{\circ}$ for the cases of one or two nonhorizontal attitudes, respectively. Pont et al. (1996) and Gordon and Morison (1982) found curvature discrimination thresholds in terms of local attitude in the order of magnitude of $1^{\circ}$. This is in the same order of magnitude as that which we found in the present investigation. The results of the first experiment show that the local attitudes on a curved surface can be determined per finger. This result suggests that, in our experiments, curvature discrimination takes place via a comparison of the local attitudes of two successively presented strips. On the other hand, the thresholds for conditions in which there are two nonhorizontal attitudes are about a factor of two smaller than those for conditions in which there is only one nonhorizontal attitude. The attitude differences over the strips are also a factor of two smaller in those cases. This suggests that subjects are able to improve performance by comparing the attitude differences over the surfaces. Apparently, comparisons both of local attitudes on the surfaces of successively presented stimuli and of attitude differences over the surfaces play a role in discrimination of curvature. The degree to which one mechanism predominates will depend on the exact conditions under which the discrimination takes place.

\section{Summary of the Results of Experiment 1}

The thresholds for the second- and first-order stimuli in units of local attitude are not systematically different, and the curvature cannot be discriminated locally. We conclude that the second-order geometrical structure, the local curvature, does not contribute markedly to discrimination of curved strips from a flat one. The base-to-peak height difference is also excluded as a cue for this discrimination task. Altogether, we conclude that the discrimination of curved strips from flat ones is based on local attitudes exclusively.

\section{EXPERIMENT 2}

This experiment examined whether the subject takes the positions of the fingers on the stimuli into account. We therefore measure points of subjective equality for curved strips that are touched over different lengths. Ex-

A

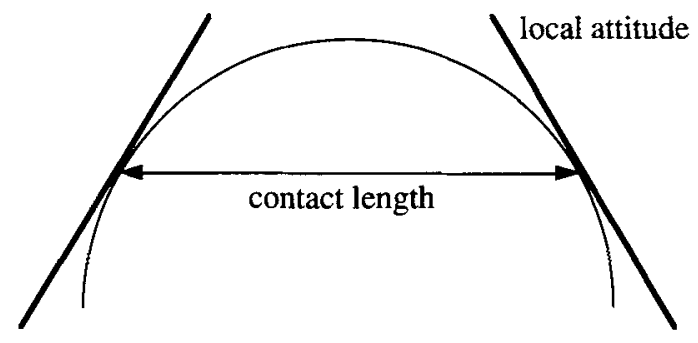

B

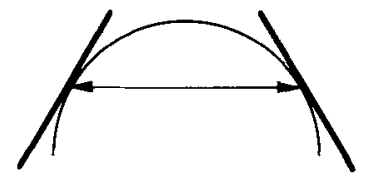

$\mathbf{C}$

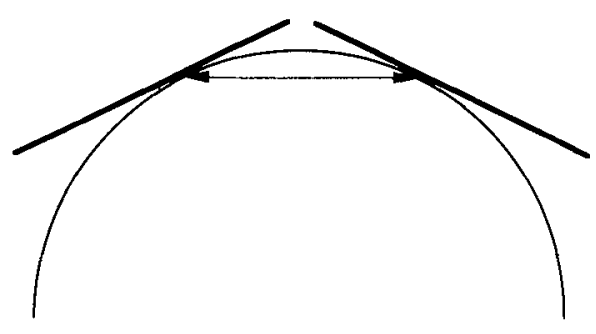

Figure 8. A schematic representation of three situations in which the fingers contact a convex curved strip. The attitude difference is the same in situations $A$ and $B$ and smaller in $C$. The curvature is the same in situations $A$ and $C$ and larger in $B$. The contact length is the same in situations $B$ and $C$ and larger in $A$. 
periment 1 showed that curvature discrimination is based on local attitudes. If a curved stimulus is touched over a certain length, the local attitudes will have certain values. If the same stimulus is touched over a larger length, the local attitudes will be larger (except the one in the middle of the strip, which is always horizontal). Thus, the larger contact length leads to better discrimination performance. This could mean that stimuli are judged to be less curved if they are touched over a smaller contact length than if they are touched over a larger contact length. In Experiment 2, we investigated whether this is the case; if curved stimuli that are touched over a smaller length are judged to be less curved, in comparison with the same stimuli that are touched over a larger length, the points of subjective equality will differ from the reference values. In that case, subjects will judge the curvatures to be the same in situations $\mathrm{A}$ and $\mathrm{B}$ of Figure 8 (because the attitude differences are the same), and they will judge the curvature to be smaller in situation $\mathrm{C}$ than in situation A (because the attitude difference is less in C). However, there is a possibility that subjects are able to correct for differences in contact lengths. In that case, the points of subjective equality will not differ from the reference values (curvature comparison will be veridical; the curvature will be judged to be the same in situations $A$ and $C$ in Figure 8, and the curvature will be judged to be higher in situation $B$ than in A).

\section{Method}

Subjects. Subjects R.B., J.B., and N.W. participated in Experiment 2. Subject H.B. was not available for this experiment.

Stimuli. We used the second-order (the curved) stimuli, which are shown on the right side of Figure 2.

Experimental setup and procedure. In Experiment 2, the reference strip was touched with the fingers held close together and the test strip with the fingers spread out wide (condition reference close), or vice versa (condition reference wide). In the wide configuration, the fingers were spread out so that the hand posture was still comfortable to the subject. In this way, we investigated whether the positions of the fingers on the stimuli are known and accounted for in the subjects' judgments of curvature. In these measurements, 3 reference strips- 1 straight, 1 convex, and 1 concave-were combined with 14 test strips. The reference strips had curvatures of $0 / \mathrm{m}$, $+2 / \mathrm{m}$, or $-2 / \mathrm{m}$, and the test strips had curvatures that differed by a maximum of $1.8 / \mathrm{m}$ from these values. Each combination was presented eight times. Both experimental conditions thus consisted of 3 (reference strips) $* 14$ (test strips) $* 8$ (presentations) $=336$ trials and were tested in four blocks of 84 trials. In each of these four blocks, we presented all the different combinations twice in the same order. The reference strips were presented first and the test strips second in the first and third block, and vice versa in the second and fourth block. The subjects did not know or notice this. Three repeated measurements were done for condition reference close with N.W., to get an indication of the reproducibility of these measurements. This experiment consisted of about $6 \mathrm{~h}$ of measurements.

\section{Results}

In Experiment 2, the reference and test strips were touched at different positions: close and wide, or vice versa. In Figure 9, we show a comparison of the thresholds for the second experiment with those for the first experiment for the second-order stimuli under condition "imr" in terms of curvature. The values for Experiment 2 are the means of conditions reference close and reference wide for the straight reference strip. The value for N.W., Experiment 1, is smaller than the stepwidth between the stimuli. This value is thus not realistic, but it is clear that performance is poorer for the discrimination of strips that were touched over differing contact lengths than for those touched over the same contact length (by a factor of about two for J.B. and R.B.). Moreover, the psychometric curves showed nonvanishing biases. Figure 10 shows the biases.

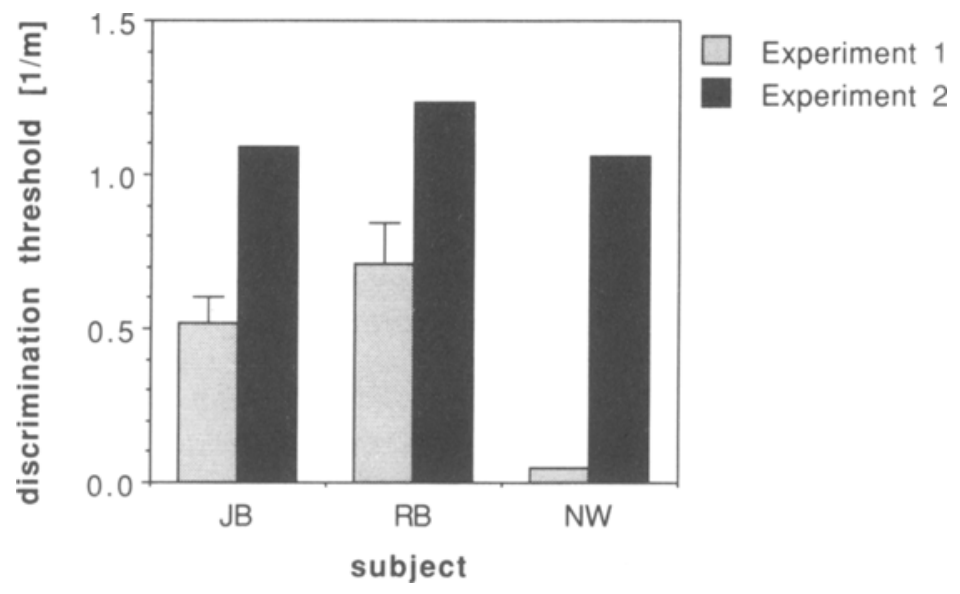

Figure 9. A comparison of the thresholds for Experiment 2 (dark bars) with those for Experiment 1 (light bars) for the second-order stimuli under condition "imr" in terms of curvature. The values for Experiment 2 are the means of conditions "reference close" and "reference wide" for the straight reference strip. On the horizontal axis are the 3 subjects who participated in Experiment 2. 

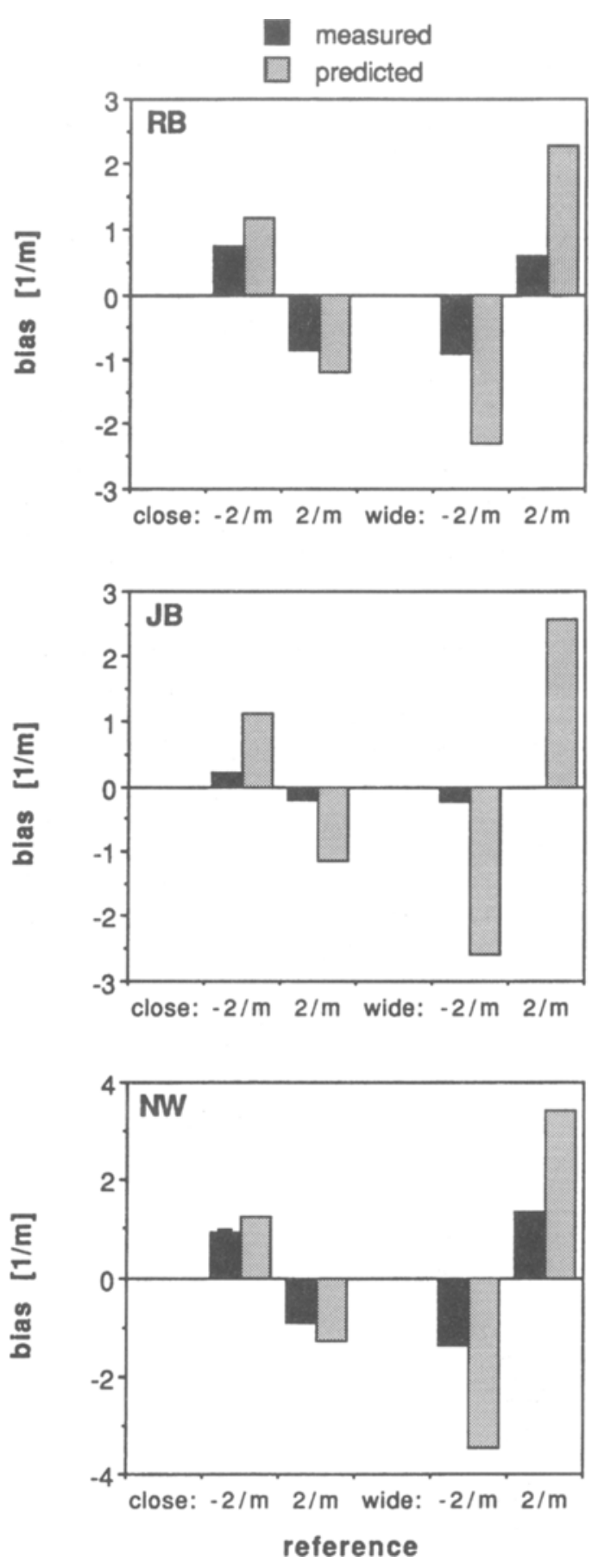

Figure 10. The biases in terms of curvature for the conditions that were tested in the second experiment. The values are for subjects R.B., J.B., and N.W. (upper, middle, and lower panels, respectively). Two values are shown for each condition: one value that was derived from the measurements (dark bars) and one that was calculated by assuming that the subjects only had knowledge about the local attitudes and relative positions of the fingers, but not about the exact distance between the fingers (light bars). The manner in which the reference curvature was touched (close or wide) and its curvature $(-2 / \mathrm{m}$ or $2 / \mathrm{m}$ ) are depicted on the horizontal axis. Standard errors were calculated to be very small for the measurements with N.W.; they are invisible for all datapoints except the most left.
The bias for reference curvature $0 / \mathrm{m}$ was subtracted from the original values for the reference curvatures $+2 / \mathrm{m}$ and $-2 / \mathrm{m}$. We did this because, for all 3 subjects and for both conditions, the values for the convex and concave references lay symmetrically around the values for the flat reference. The bias at $0 / \mathrm{m}$ probably represents a response bias, which might be caused by the method of testing the different orders (first reference strip-second test strip, and vice versa) in different blocks of measurements. For subjects R.B., J.B., and N.W., we show the biases that were measured, as well as those that were calculated by assuming that the subjects ignored the distance between the fingers and used only the total difference of local surface attitude over the touched part of the surface (if the subjects correct for contact length differences veridically, the biases will, of course, be zero). For N.W. in condition reference close, the standard errors are indicated and clearly are very low. The biases that were measured are lower than those that were calculated, but certainly not zero (except for one value for J.B.), and they are in the same direction as those calculated. These results show that, to some extent, the subjects do take the different contact lengths into account, but they make systematic errors. These errors suggest that the larger contact lengths are underestimated, relative to the smaller ones.

\section{Discussion}

Curvature discrimination cannot be based on local attitudes only. The attitudes have to be combined with some knowledge about the locations of these attitudes. This knowledge might consist of the exact positions of the fingers, relative to each other. Another possibility is that only rough knowledge is taken into account-for instance, the ring finger is on the right of the middle finger and the index finger on the left. In the second experiment, in which the strips were touched over varying contact lengths, we found that the subjects made systematic errors in their judgments of the curvature of these stimuli. Furthermore, the discrimination thresholds increased by a factor of about two under conditions of varying contact lengths. Durlach et al. (1989) and Tan, Pang, and Durlach (1992) investigated haptic length perception and did not find systematic overestimations or underestimations for length perception by means of the finger-span method. However, it is quite difficult to extrapolate their results to ours, because the manner in which the stimuli were touched and their experimental task were different. In the present investigation, the positions of the fingers were changed in the direction at right angles to the fingers and along the strips. The local attitudes also changed along the curved strips. These variations in contact length resulted in systematic errors in curvature discrimination. It is, therefore, unlikely that the exact positions of the fingers were taken into account in the judgments of the curvature of the stimuli.

Thus, our results indicate that curvature discrimination takes place via a mechanism in which the local attitudes are combined with rough knowledge of the positions of the fingers. The thresholds for curvature discrimination in terms of attitudes were of the order of $0.8^{\circ}-5^{\circ}$, which 
are very small values. Thus, the finger positions, relative to the strips, must have been known or been the same during a trial, up to a high accuracy. But the exact positions of the fingers are not taken into account in the judgments of the curvature of the stimuli. Taken together, these conclusions suggest that the similarity of the finger positions or hand posture during a trial provides the basis for the comparison of the local attitudes of two successively presented strips. This raises important questions: How does the final combination of local attitudes and their locations take place? Gardner (1988) described "haptic neurons" that integrate tactile and kinesthetic stimulations. She found that these specific neurons code global features at the expense of a loss of information concerning finegrained spatial detail. It might be that something similar is happening in our task.

\section{Summary of the Results of Experiment 2}

The discrimination thresholds are larger if the strips are touched over different lengths than if they are touched over the same lengths. The subjects judge a strip to be more curved if it is touched over longer lengths than if it is touched over shorter lengths. We conclude that subjects probably do not take the exact positions of the fingers into account in judgments of the curvature of the strips.

\section{EXPERIMENT 3}

In this experiment, we investigated whether the discrimination thresholds for dynamic curvature discrimination vary as a function of stimulus length. In addition, we analyzed whether it was possible to indicate the effective stimulus for dynamic curvature discrimination. If dynamic curvature discrimination is based on comparison of curvatures (attitude differences, height differences), the thresholds in terms of curvature (attitude difference, height difference), will be constant (see Pont et al., 1997).

\section{Method}

Subjects. Three naive subjects participated in the experiments. They were paid for their efforts. The subjects were unaware of the objectives of the experiment. Subjects I.L. and F.K. are strongly right-handed; M.B. is ambidextrous (according to definitions used by Coren, 1993).

Stimuli. We used the second-order strips (which are depicted in Figure 2, on the right side). The length over which the strips were touched was varied by means of four windows that were placed in front of the strips (with respect to the subject). These windows were openings in which the subject could touch the strips with the index finger in a dynamic way (Figure 11A). The windows were 5, 10, 15, or $20 \mathrm{~cm}$ long (Figure 11B). The middle of the strips was always located in the middle of the windows.

Experimental setup and procedure. The setup was the same as that in the former experiments. The strips were now touched in a dynamic way with the index finger of the right hand, meaning that the subjects put their index finger on and moved their finger to and fro over the curved surfaces. The subjects were told to move their index finger over the strips from the left to the right edges of the windows, and vice versa, without stopping. Thus, the stimulus
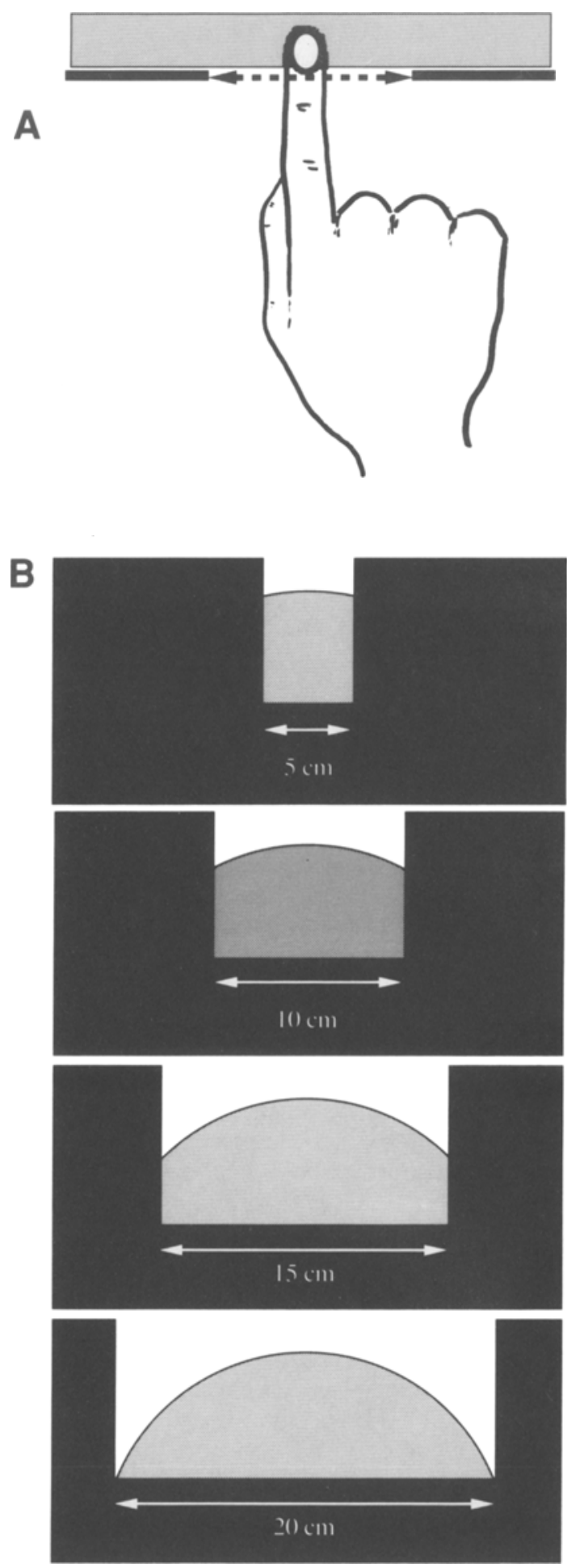

Figure 11. Schematic representation of the experimental conditions in Experiment 3. Panel A represents a top view of an index finger of a right hand touching a strip (in gray) through a window (in black). The finger can be moved to and fro over a strip length that is controlled by means of the window. Panel $B$ represents the four different stimulus lengths, which are specified by means of the windows (in black). For clarity, the strips (in gray) are depicted with much larger curvatures than they have in reality. Only convex stimuli are shown. 
lengths were well defined by the lengths of the window openings. The strips and windows were fixed in a frame so that they were always presented in the same place: at right angles to the subject's finger, under the distal phalanx of the index finger. The positions of the stimuli were checked by the experimenter.

The task of the subjects was to touch two stimuli successively and to indicate which of the two strips was the more convex. We made sure that the subjects did not have difficulties with this concept. Before the first test, they did some trials on combinations of 20 -cm stimuli with the largest curvatures used. Feedback was given on these trials, and a session was started after the subjects had correctly judged four of these combinations successively. No feedback was given during the actual experiments.

We tested discrimination performance under four conditions. In these conditions, both stimuli were touched over $5,10,15$, or $20 \mathrm{~cm}$. The subjects were told to move their index finger twice to and fro over the stimulus surface. The experimenter checked the number of movements the subjects made.

In each condition, a flat reference strip was combined with 14 test strips, 7 convex and 7 concave stimuli. Because the discrimination thresholds differed for the different conditions, the measuring range was adjusted after each test, on the basis of the performance in the previous test. All the combinations were presented eight times: four times in one order (first the reference and second the test strip) and four times in the other order (first the test and second the reference strip) in a random sequence. Each experimental condition thus consisted of 14 (test strips) $* 8$ (presentations) $=$ 112 trials and was tested three times on different days. The different conditions were tested in a random order. This experiment consisted of about $16 \mathrm{~h}$ of measurements.

Analysis. Cumulative Gauss distributions were fitted as psychometric curves in the same manner as that in Experiment 1. We again calculated the means and the standard errors over the parameters of the separate curves for each of the three measurements per condition.

\section{Results}

The means, or points of subjective equality, for the third experiment do not differ systematically from the reference values. This was expected, because possible influences of biases were counterbalanced in this experiment.

The discrimination thresholds as a function of the stimulus length are presented in Figure 12 (the unit is curvature). The results for F.K., M.B., and I.L. are presented in the upper, middle, and lower panels, respectively. It is clear that the thresholds decrease for longer stimulus lengths, up to a certain degree: The difference between the thresholds for the 5 - and $10-\mathrm{cm}$ stimuli is very clear, the difference for the 10- and 15-cm stimuli is smaller but still clear, and the difference between the 15- and $20-\mathrm{cm}$ stimuli is not clear. Thus, the curvature that is needed to discriminate a curved strip from a flat one decreases for longer strips up to about 15 or $20 \mathrm{~cm}$.

We also calculated the thresholds and standard errors in terms of the radius of curvature, the attitude difference, and the base-to-peak height difference over the surface; these are depicted in Figure 13 in the left, middle, and right panels, respectively (note the redundancy of these figures; they give a complete overview of the results, but, of course, the left, middle, and right panels are different views of the same data as those presented in Figure 12). The upper, middle, and lower panels show the discrimination thresholds for F.K., M.B., and I.L., respectively. The
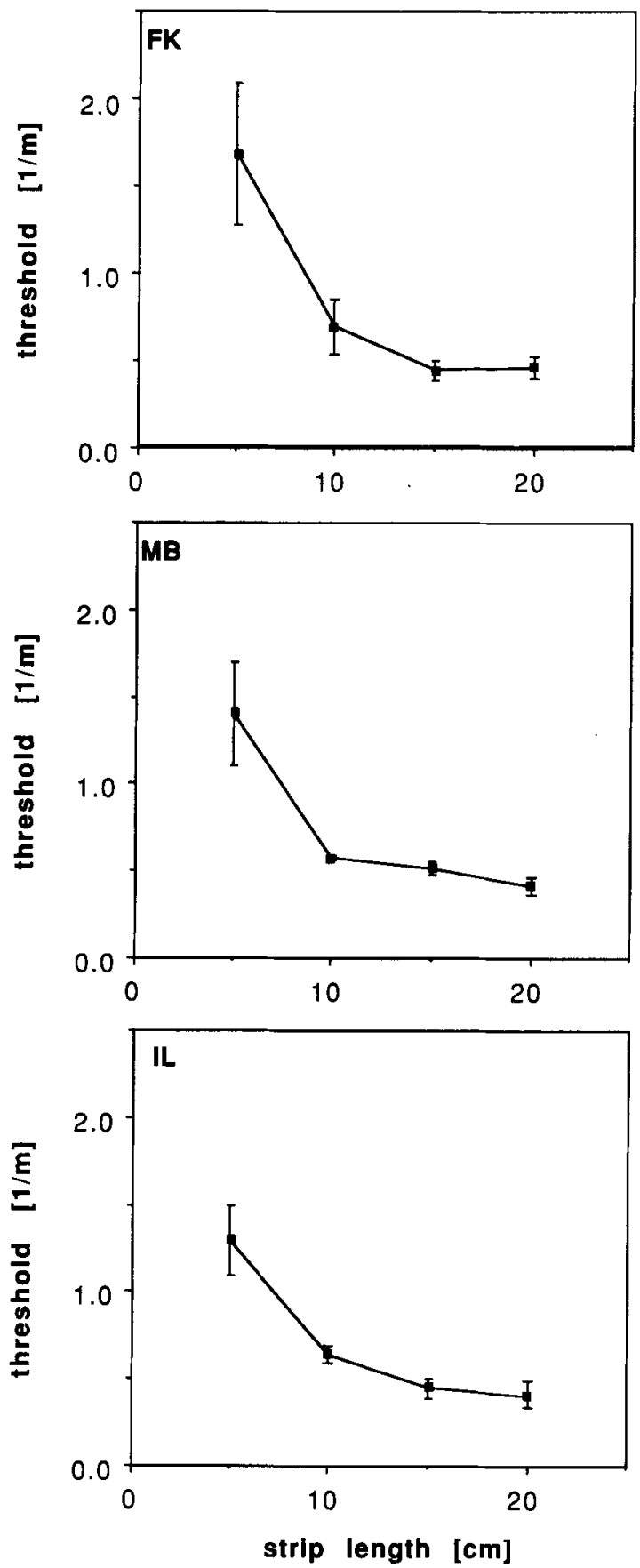

Figure 12. The discrimination thresholds as a function of strip length for F.K., M.B., and I.L. measured in Experiment 3.

solid lines represent linear regressions on the data, which were calculated, by means of the least squares method, from the separate measurements (i.e., 4 datapoints $* 3$ measurements $=12$ thresholds) in order to take the variances into account. It is clear that the thresholds expressed in terms of radius of curvature and base-to-peak height difference (Figure 13, left and right panels) increase as a function of stimulus length. So, the radius of curvature or the base-to-peak height difference that is needed to dis- 

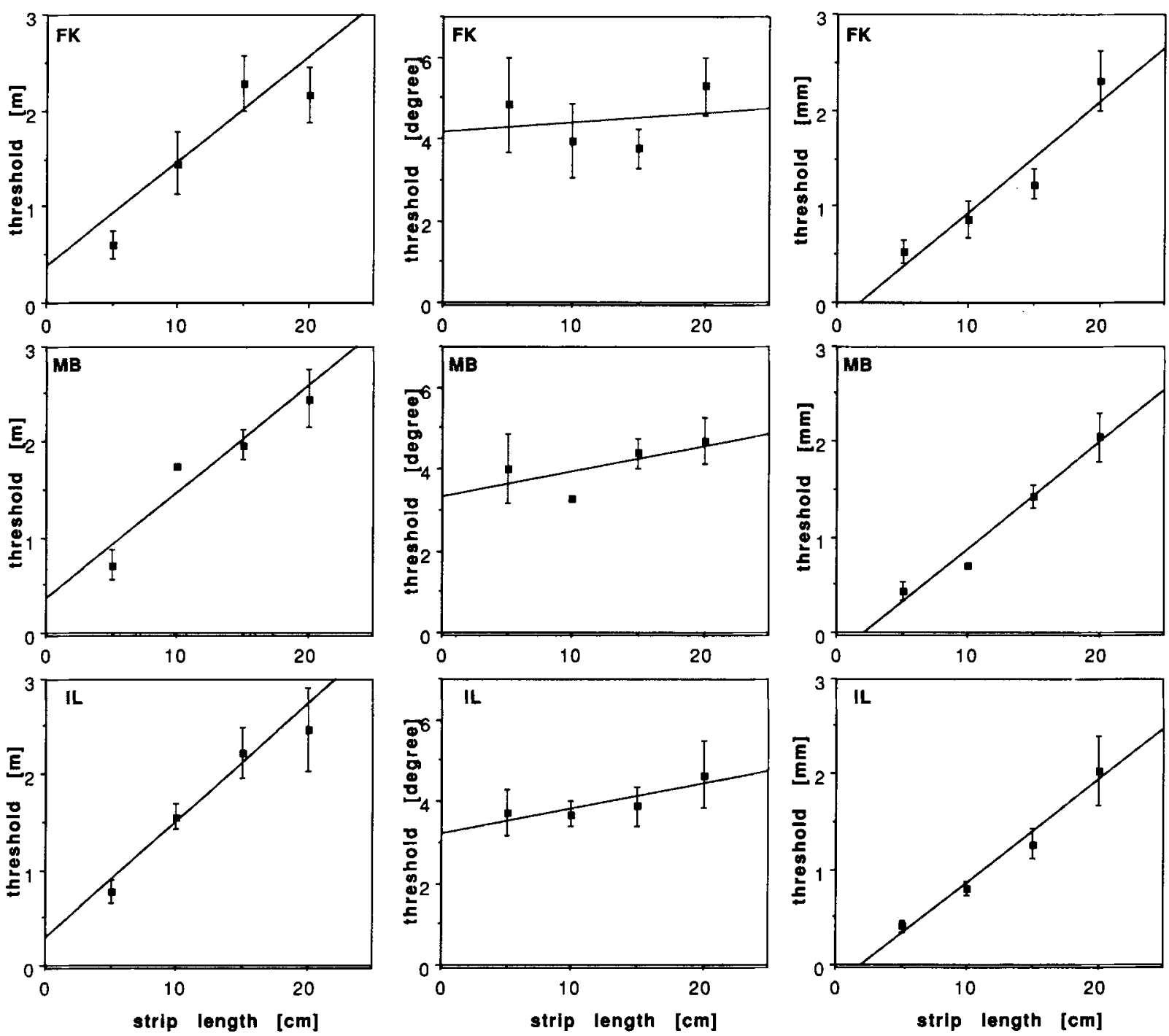

Figure 13. The diserimination thresholds expressed in terms of radius of curvature (left panels), attitude difference (middle panels), and base-to-peak height difference (right panels) as a function of strip length.

criminate a curved strip from a flat strip increases with longer strips. The thresholds expressed in terms of the attitude difference (Figure 13, middle panels) are almost constant; one-tailed $t$ tests showed that the slopes of the regressions on these data were not significantly different from $0(p>.10)$.

\section{Discussion}

It should be noted that we analyzed the discrimination thresholds in terms of the base-to-peak height difference and the total attitude difference over the surface. If one thinks of the possible mechanisms that underlie dynamic curvature discrimination, one would not expect height or attitude differences to be determined at the extreme contact points on the stimulus (because the centers of the contact areas are a little displaced, relative to the extreme contact points on the stimulus). It seems that some kind of average or integrated value is more appropriate for describing possible mechanisms underlying dynamic touch. However, the influence of such aspects on our analysis is very slight and not of decisive importance for this analysis.

The thresholds expressed in terms of radius of curvature or base-to-peak height difference increase as a function of stimulus length. The radius of curvature or height difference that gives rise to threshold level performance must, therefore, be larger for longer stimuli. If curvature discrimination is based on the comparison of radii of curvature or height differences, the radius of curvature (and its reciprocal, the curvature) or the height difference at threshold level will be constant. It must, therefore, be concluded that the radius of curvature or the height difference is not the effective stimulus for curvature discrimination in this experiment. The discrimination thresholds, expressed in terms of the attitude difference over the surface, 

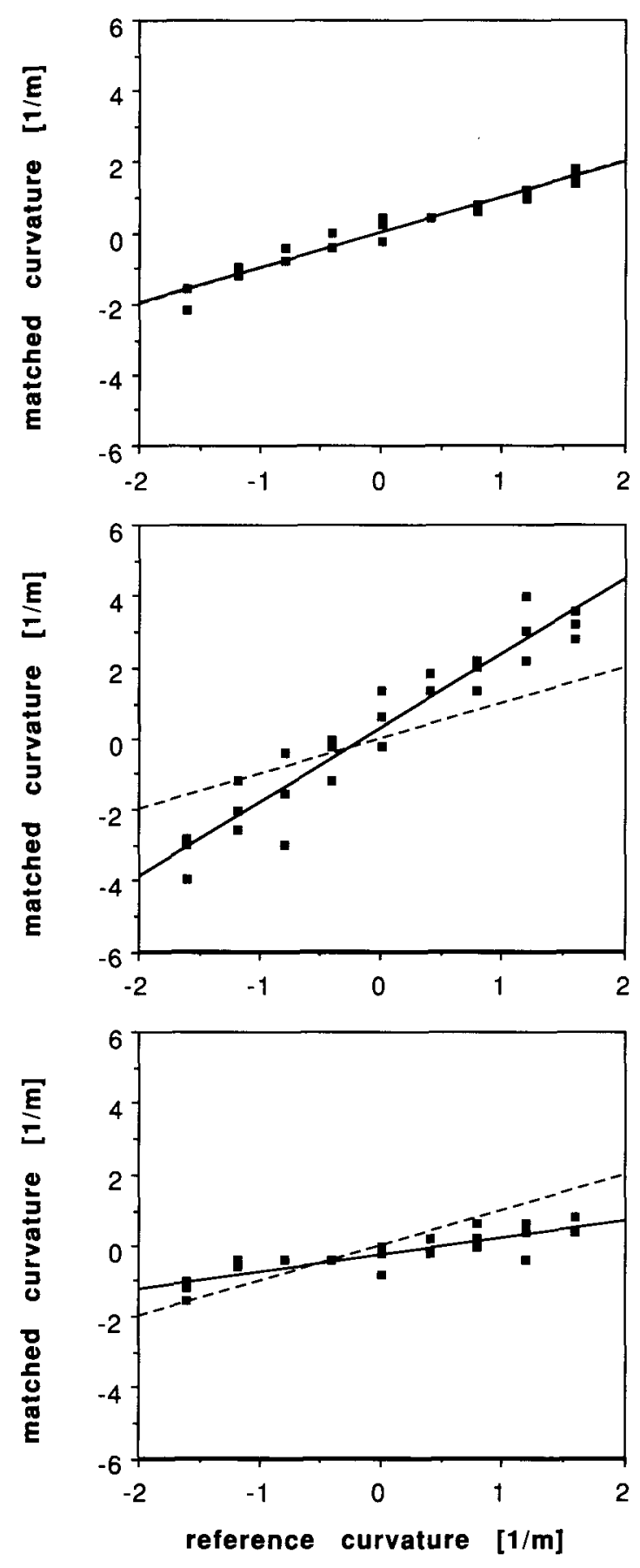

Figure 14. Three typical examples for the rough datapoints and the linear functions fitted through them from the matching experiment (subject F.K., Experiment 4). The curvature of the reference strip is on the horizontal axis; the curvature of the test strip is on the vertical axis. The upper panel shows an example for matching strips of the same length (condition 20-20; fitted function, $y=.02+.99 x$, and $r^{2}=.96$ ), the middle panel for a case in which the reference strip was shorter than the test strip (condition 5-20; fitted function, $y=.32+2.09 x$, and $r^{2}=.91$ ), and the lower panel for a case in which the reference strip was longer than the test strip (condition 20-5; fitted function, $y=$ $-.19+.49 x$, and $\left.r^{2}=.75\right)$. do not increase significantly as a function of stimulus length. This is what one expects if comparison of local attitude differences underlies curvature discrimination: The attitude difference needed to reach threshold level performance is independent of the length of the stimulus. In this experiment, therefore, curvature discrimination seems to be based on the comparison of attitude differences.

To summarize, in our experiment, curvature discrimination does not take place via the direct comparison of curvatures or via the comparison of height differences. It is the first-order geometrical structure (the attitude difference) that seems to represent the effective stimulus for curvature discrimination in this experiment. Gordon and Morison (1982) also came to this conclusion when they studied dynamic discrimination of curved surfaces of $1 \times$ maximally $4 \mathrm{~cm}$ with the index finger. They found a gradient of .009 at $75 \%$ correct, which corresponds to an attitude difference of $2.8^{\circ}$ over the surface at $84 \%$ correct. The thresholds we find are in the range $3.3^{\circ}-5.3^{\circ}$ and, thus, are about a factor of two larger. Gordon and Morison showed that performance in their experiments was better than that in experiments in which larger (than $1 \times 4 \mathrm{~cm}$ ) curved surfaces were used and larger scanning movements, involving the forearm, were made. The poorer performance in our experiment might also be explained by the difference in stimulus dimensions and scanning movements; our subjects moved their forearms while scanning the surfaces of $2 \times 5-20 \mathrm{~cm}$. A rough comparison between our data and Davidson's (1972) for the condition that was closest to our conditions (the condition in which vertical curves of $1.9 \times 20 \mathrm{~cm}$ were judged and the movements involved the forearm) showed that the sensitivity was of the same order of magnitude.

\section{Summary of the Results of Experiment 3}

In Experiment 3, the local curvature and the base-topeak height difference were excluded as the basis for dynamic curvature comparison. The first-order geometrical structure (the attitude differences) seems to represent the effective stimulus.

\section{EXPERIMENT 4}

In Experiment 3, we found that curvature discrimination performance was poorer for shorter than for longer stimuli. This could mean that shorter stimuli with a certain curvature are judged to be less curved than longer stimuli with the same curvature. In Experiment 4, we investigated whether this was the case: If subjects judge shorter stimuli to be less curved than longer stimuli with the same physical curvature, they will make systematic errors in the conditions in which stimuli of different lengths are required to be matched (e.g., the curvature will be judged to be less in situation $\mathrm{C}$ than in A in Figure 8). However, there is a possibility that, in their judgments of the curvatures of the stimuli, subjects are able to correct for different stimulus lengths. In that case, they will match curvature veridically under all experimental conditions (e.g., 
the curvature will be judged to be the same in situations $\mathrm{A}$ and $\mathrm{C}$ in Figure 8).

\section{Method}

Subjects. The subjects who participated in Experiment 3 also participated in Experiment 4.

Stimuli. The haptic stimuli were the same curved strips as those used in the former experiments (Figure 2, on the right). The length over which the strips were touched was again varied by means of the windows that were placed in front of the strips (with respect to the subject). The windows used in Experiment 2 were 5,10 , or $20 \mathrm{~cm}$ long (Figure 11). The middle of the strip was always located in the middle of the window.

Experimental setup and procedure. The experimental setup was the same as that in the former experiments. The procedure differed only in the task and in the fact that the experimenter wrote down the matched curvatures. The strips were touched dynamically with the index finger. The task of the subjects was to touch a strip (called the reference) and to match its curvature with the curvature of another strip (called the test), which was presented successively and in the same place. After the first presentation of the reference and test strips, the subject could ask for the reference or another test strip and indicate whether this test strip was to have a higher or lower curvature than the test strip presented first. This procedure was repeated until the subject indicated that he or she was sure that the curvatures of the reference and the test strips were the same. Then the experimenter wrote down which test strip the subject judged to have the same curvature as the reference strip. The subjects were not restricted in the number of times they touched or the number of movements they made over the reference and test strips; they could ask for the reference and test strips as often as they wanted and until they felt sure about their judgment. The only restriction was that they had to move their index fingers over the surfaces over the full length of the windows. Of course, the subjects did not get feedback during this experiment.

We tested nine conditions, which consisted of all nine possible combinations of reference and test stimuli 5,10 , or $20 \mathrm{~cm}$ in length. So, we tested three combinations of reference and test strips of the same lengths, three combinations of reference strips that were longer than the test strips, and three combinations of reference strips that were shorter than the test strips.

In these measurements, the reference strips had curvatures of $-1.6 / \mathrm{m},-1.2 / \mathrm{m},-0.8 / \mathrm{m},-0.4 / \mathrm{m}, 0 / \mathrm{m}, 0.4 / \mathrm{m}, 0.8 / \mathrm{m}, 1.2 / \mathrm{m}$, and $1.6 / \mathrm{m}$. Each reference strip was presented three times. The reference and test strips that were presented first had curvatures that differed by $-0.4 / \mathrm{m}, 0 / \mathrm{m}$, or $+4 / \mathrm{m}$. The subjects could choose test strips with curvatures ranging from $-4 / \mathrm{m}$ to $+4 / \mathrm{m}$, with a stepwidth of $0.2 / \mathrm{m}$. All the experimental conditions consisted of 9 (reference strips) * 3 (presentations) $=27$ trials. Experiment 4 thus consisted of 9 (conditions) $* 27$ (trials) $=243$ trials, which were tested in a random order. The 243 trials were divided into three blocks of 81 trials. The measurement of the first (second, third) block was done after the first (second, third) series of measurements of Experiment 3. The measurements of Experiments 3 and 4 were alternated, because we wanted to compare the results and because the alternation of discrimination and matching experiments was more convenient for the subjects. We measured in 27 sessions of about $1 \mathrm{~h}$.

\section{Results}

The matched curvatures were plotted as a function of the reference curvature. We fitted linear functions to these datapoints by means of the least squares method. In Figure 14, we show three typical examples of datapoints and the linear functions fitted through them. The reference curvature is on the horizontal axes, and the matched cur-
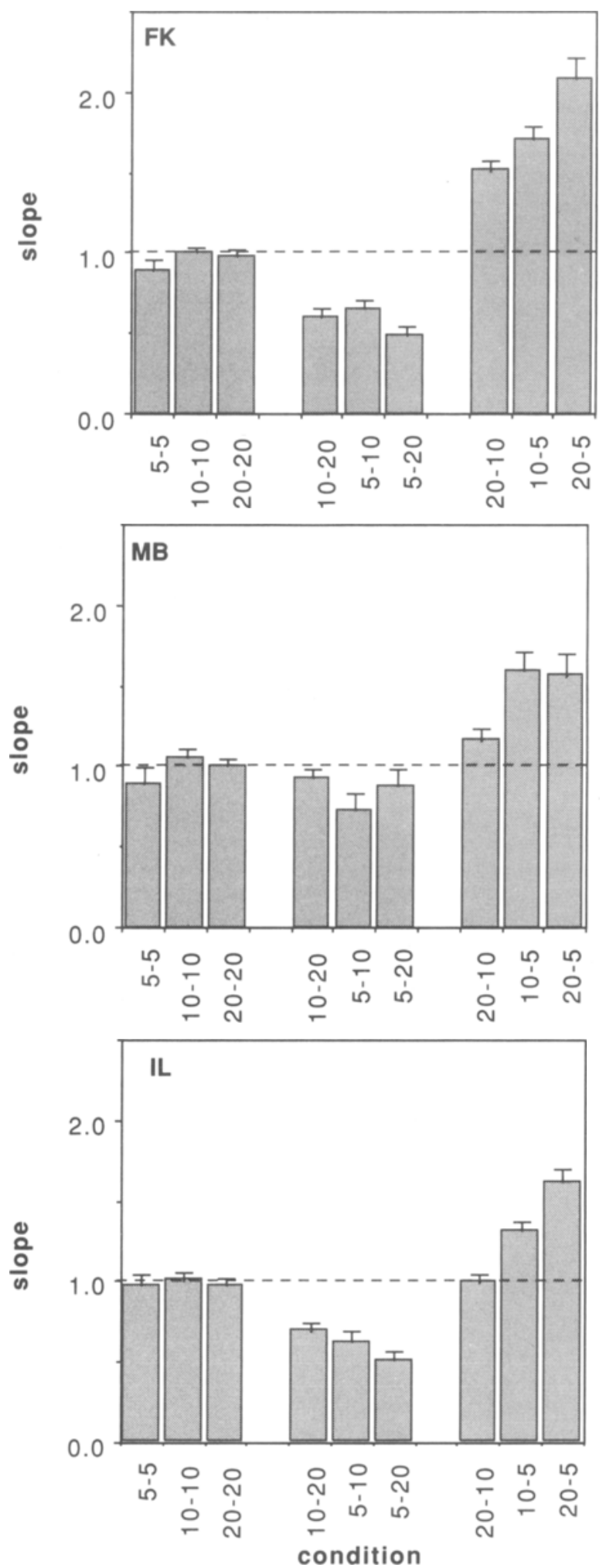

Figure 15. The slopes that were determined from the matching data. The upper, middle, and lower panels show the data for F.K., M.B., and I.L., respectively. The conditions are on the horizontal axes: The first value is the length (in centimeters) of the reference strip; the second is the length of the test strip. 
vature is on the vertical axes. The upper, middle, and lower panels show the data for conditions reference $20 \mathrm{~cm}$ and test $20 \mathrm{~cm}$, reference $20 \mathrm{~cm}$ and test $5 \mathrm{~cm}$, and reference $5 \mathrm{~cm}$ and test $20 \mathrm{~cm}$, respectively, for subject F.K. The fitted functions are characterized by a slope and an offset. In the case of veridical curvature matching, the slope is 1 , and the offset is 0 (the dotted lines in Figure 14). The slope is the most interesting parameter. A slope larger (smaller) than 1 will be found if the curvatures of the test stimuli are underestimated (overestimated), as compared with the curvature of the reference stimuli. Figure 14 shows one case of veridical curvature matching (upper panel), one of underestimation (middle panel), and one of overestimation (lower panel) of the absolute curvatures of the test as compared with the reference stimuli. The linear correlation of the data is represented by the correlation coefficient $r^{2}$.

The resulting slopes for the 3 subjects for the matching experiment are depicted in Figure 15. The combinations of strip lengths are on the horizontal axes; the first value is the length (in centimeters) of the reference strip, the second of the test strip. The slopes are shown on the vertical axes. In the cases of strips with the same lengths (conditions 5-5, 10-10, 20-20), the slopes are about equal to 1 , meaning that the curvatures are matched veridically. In conditions 10-20,5-10, and 5-20, the matched test strip was longer than the reference strip. Here we find slopes smaller than 1 , meaning that the absolute curvature of the longer test strip was overestimated, as compared with the shorter reference strip. This effect is very large: In two cases (conditions 5-20 for subjects F.K. and I.L.), we even find a slope of .5 , which means that the curvature of the $20-\mathrm{cm}$ strip is overestimated, as compared with the 5 -cm strip, by a factor of 2 . In the case of a reference that is longer than the matched stimulus (conditions 20-10, $10-5,20-5$ ), the slope is larger than 1 (with one exception: subject I.L., condition 20-10), meaning that the absolute curvature of the shorter test strip is underestimated, as compared with that of the longer reference strip. This effect is also very large: The maximum value of the slope is 2.1 (condition $20-5$ for subject F.K.), meaning that the curvature of the 5 -cm strip was underestimated, as compared with the $20-\mathrm{cm}$ strip, by a factor of 2.1 . From comparisons of the slopes for conditions $10-20$ and 5-20 or 20-10 and 20-5, it can also be seen that the deviation of the slope from 1 is larger if the difference between the lengths of the strips is larger. However, in three cases, the results for conditions 5-10 and 10-5 are not consistent with this trend (subject F.K., condition 5-10; subject M.B., conditions 5-10 and 10-5). To test whether the deviations of the slopes from 1 were significant, we performed $t$ tests. Table 1 , second column, shows the numerical values of the slopes and their significance levels for the nine conditions and the 3 subjects. It can be seen that the slopes do not differ significantly from 1 in most of the cases in which two stimuli of the same length were matched. If the lengths of the strips differ, all the slopes except three are significantly different from 1 .
In Table 1, we also show the numerical values of $r^{2}$ (fourth column), the offsets and the significance levels of the offsets that were determined via $t$ tests (third column). It can be seen that the correlation coefficient $r^{2}$ is, overall, quite high. The lowest values are found for cases in which strips with a length of $5 \mathrm{~cm}$ were matched (for instance, subject F.K., condition 5-20; subject M.B., condition 5-10; subject I.L., condition 5-10). Since the data did not show deviations from linear relations, it is therefore possible that these differences in the $r^{2}$ reflect, to some extent, the accuracy with which the judgments were made. Most offsets differ significantly from 0 , but not in a systematic way.

\section{Discussion}

We found that the curvature of a longer strip is overestimated, as compared with that of a shorter strip. Thus, subjects make systematic errors in the matching of the curvatures of strips of different lengths. We will now discuss the magnitudes of these errors by making comparisons with predictions that were made on the basis of the attitude difference and attitude difference and contact length correction models described earlier.

In Figure 16, we show predictions for the slopes on the basis of the two limit cases for the processing of the geometrical structure. For the first prediction, we assume that two strips are judged to have the same curvature if

Table 1

Results of Experiment 4

\begin{tabular}{|c|c|c|c|c|}
\hline Subject & Condition & Slope & Offset & $r^{2}$ \\
\hline F.K. & $\begin{array}{l}5-5 \\
10-10 \\
20-20 \\
10-20 \\
5-10 \\
5-20 \\
20-10 \\
10-5 \\
20-5\end{array}$ & $\begin{array}{l}0.90 \\
1.00 \\
0.99 \\
0.60 \dagger \\
0.65 \dagger \\
0.49 \dagger \\
1.51 \dagger \\
1.71 \dagger \\
2.09 \dagger\end{array}$ & $\begin{array}{c}-.10 \\
.07 \\
.02 \\
-.15^{*} \\
-.08 \\
-.19^{*} \\
.25^{*} \\
.13 \\
.32^{*}\end{array}$ & $\begin{array}{l}.89 \\
.97 \\
.96 \\
.84 \\
.83 \\
.75 \\
.95 \\
.95 \\
.91\end{array}$ \\
\hline M.B. & $\begin{array}{l}5-5 \\
10-10 \\
20-20 \\
10-20 \\
5-10 \\
5-20 \\
20-10 \\
10-5 \\
20-5\end{array}$ & $\begin{array}{l}0.89 \\
1.05 \\
1.01 \\
0.94 \\
0.73^{*} \\
0.88 \\
1.16^{*} \\
1.60^{\dagger} \\
1.5 \dagger^{\dagger}\end{array}$ & $\begin{array}{c}.48 \dagger \\
-.02 \\
.04 \\
-.13^{*} \\
.59 \dagger \\
.24^{*} \\
.07 \\
-.53 \dagger \\
-.54 \dagger\end{array}$ & $\begin{array}{l}.74 \\
.93 \\
.97 \\
.94 \\
.68 \\
.73 \\
.91 \\
.88 \\
.85\end{array}$ \\
\hline I.L. & $\begin{array}{l}5-5 \\
10-10 \\
20-20 \\
10-20 \\
5-10 \\
5-20 \\
20-10 \\
10-5 \\
20-5\end{array}$ & $\begin{array}{l}0.98 \\
1.00 \\
0.97 \\
0.69 \dagger \\
0.62 \dagger \\
0.51 \dagger \\
0.99 \\
1.31 \dagger \\
1.61 \dagger\end{array}$ & $\begin{array}{c}-.01 \\
-.01 \\
.06 \\
.04 \\
.07 \\
-.01 \\
-.11^{*} \\
-.01 \\
.13\end{array}$ & $\begin{array}{l}.93 \\
.94 \\
.96 \\
.90 \\
.71 \\
.81 \\
.92 \\
.95 \\
.95\end{array}$ \\
\hline
\end{tabular}

Note-The second and third columns for each subject show the slopes plus their significance levels (null-hypothesis, slope equal to 1) and the offsets plus their significance levels (null-hypothesis, offset equal to 0 ), respectively. The significance levels were determined by means of $t$ tests (two-tailed). ${ }^{*} p<.05 . \quad{ }^{\dagger} p<.001$. 


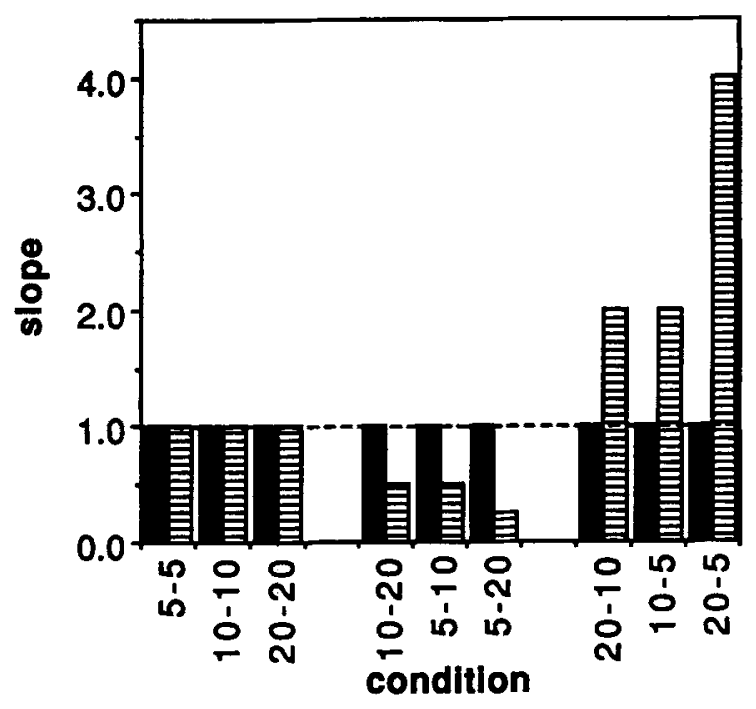

Figure 16. The predictions for the slopes in the case of comparison of attitude differences with veridical correction for contact length differences (black bars) or no correction for contact length differences (striped bars). On the horizontal axis are the conditions or length combinations of the reference strip (first value) and test strip (second value).

they have the same attitude difference, irrespective of the strip lengths. For this model, we neglected such possible factors as the exact manner in which the attitude differences are determined (by means of, for instance, some kind of average or integration). These factors would only be of minor influence on the predictions. If the contact length differences are corrected for veridically (second limit case), the matching will be veridical and, therefore, all the slopes are equal to 1 (black bars in Figure 16). If attitude differences are compared (striped bars in Figure 8), the slopes will be the same as the ratio between the lengths of the strips in the first order approximation: 1 (conditions 5-5, 10-10, and 20-20), $1 / 2$ (conditions 10-20 and 5-10), 1/4 (condition 5-20), 2 (conditions 20-10 and 10-5), and 4 (condition 20-5). In Experiment 3 , we found that an attitude-comparison model fits the data. The experimentally determined slopes in Experiment 4 (Figure 15) show deviations from 1 in the same directions as those predicted on the basis of the attitude-comparison model, in which contact length differences are ignored, but the deviations are not as large as those predicted. Thus, the judgments are not completely independent of strip length.

Virsu (1971) tested visual perception of the curvature of arcs of varying length and orientation in comparison with that of circles. He found an effect similar to ours: The curvatures of the arcs were underestimated in all cases, and the error was a decreasing exponential function of arc length. We think that this effect is visible in Figure 11B.

\section{Summary of the Results of Experiment 4}

The subjects judged longer strips to be more curved than shorter strips with the same physical curvature. Subjects do not take the exact length of the strips into account in judgments of the curvature of the strips.

\section{GENERAL DISCUSSION}

In Experiments 1 and 2, the static discrimination of curved strips in the range $-4 / \mathrm{m}$ to $+4 / \mathrm{m}$ takes place via a comparison of the local attitudes or the attitude differences over the surfaces of successively presented stimuli. Discrimination performance is best if the finger positions are similar for successively touched strips; self-controlled changes in the finger positions result in systematic errors in curvature discrimination.

Experiment 3 demonstrated that, in the case of dynamic touch, the curvature discrimination thresholds decrease as a function of strip length and that the effective stimuli for dynamic discrimination of curvature from flatness for strips of $2 \times 5-20 \mathrm{~cm}$ are attitude differences. Experiment 4 showed that subjects underestimate the curvature of shorter strips when they are compared with longer strips. These three results agree with each other: They can all be explained in terms of an attitude-comparison mechanism. The systematic errors in the results of Experiment 4 were not as large as was predicted by this mechanism, and, therefore, it must be concluded that curvature judgments are not completely independent of stimulus length.

We conclude that the results for static and dynamic curvature comparison in the range $-4 / \mathrm{m}$ to $+4 / \mathrm{m}$ are qualitatively the same. All the data can be described by an attitude-comparison model and a noncomplete correction for stimulus length. It would be interesting to see whether static and dynamic curvature comparison is also quantitatively the same. For this purpose, we will also use the large data set for static discrimination that was presented in Pont et al. (1997), because these data extend over a larger length range than the data measured in the present Experiments 1 and 2.

Figure 17 shows a quantitative comparison of the discrimination thresholds for the present and the earlier investigations. The contact length (horizontal axis) was defined in earlier studies as the distance between the centers of the outermost contact areas of the fingers and the strips. The discrimination thresholds (vertical axis) for dynamic and static touch are represented by filled circles and open squares, respectively. Figure 17 shows, surprisingly, that the sensitivity is in the same range for dynamic and static haptic curvature discrimination. One can have some reservation regarding this conclusion, since the comparison was made between results of different subjects. Altogether, we conclude that, in our experiments, similar mechanisms underlie static and dynamic haptic curvature discrimination.

Kappers and Koenderink (1996) compared the data from their experiment under conditions of active dynamic touch with data from other authors' experiments on passive static touch. They found that performance in their experiment was better, owing to the combined effects of a larger surface area and active (instead of passive) discrimination. The importance of self-controlled exploratory finger movements is stressed by several investigators 


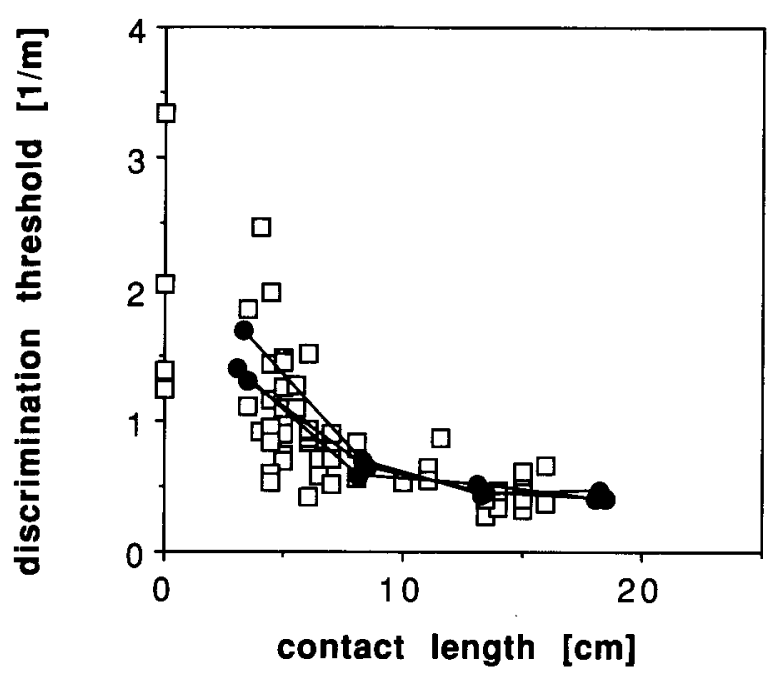

Figure 17. Comparison of the data on dynamic curvature discrimination from Experiment 3 of the present study (filled circles that are connected by lines; subjects F.K., M.B., and I.L.) and from those experiments in static curvature discrimination (open squares; subjects S.P. [not the author], I.H., and R.B.) reported in Pont et al. (1997).

(Gibson, 1962; Lederman \& Klatzky, 1987; Loomis \& Lederman, 1986). On the basis of those studies, we expected to find that dynamic haptic curvature discrimination would be superior to static haptic curvature discrimination. However, in our experiments we find (perhaps surprisingly) qualitatively and quantitatively similar results for static and dynamic touch.

\section{REFERENCES}

Clark, F. J., \& HoRCH, K. W. (1986). Kinesthesia. In K. R. Boff, L. Kaufman, \& J. P. Thomas (Eds.), Handbook of perception and human performance (pp. 13.1-13.62). New York: Wiley.

COREN, S. (1993). The left-hander syndrome. New York: Vintage Books.

Davidson, P. W. (1972). Haptic judgments of curvature by blind and sighted humans. Journal of Experimental Psychology, 93, 43-55.

Davidson, P. W., \& Whitson, T. T. (1974). Haptic equivalence matching of curvature by blind and sighted humans. Journal of Experimental Psychology, 102, 687-690.

Durlach, N. I., Delhorne, L. A., Wong, A., Ko, W. Y., Rabinowitz, W. M., \& Hollerbach, J. (1989). Manual discrimination and identification of length by the finger-span method. Perception \& Psychophysics, 46, 29-38.

FERRELl, W. R., \& Craske, B. (1992). Contribution of joint and muscle afferents to position sense at the human proximal interphalangeal joint. Experimental Physiology, 77, 331-342.

GaNdeVIA, S. C., MCCloskey, D. I., \& BURKE, D. (1992). Kinaesthetic signals and muscle contraction. Trends in Neurosciences, 15, 62-65.

GARDNER, E. P. (1988). Somatosensory cortical mechanisms of feature detection in tactile and kinesthetic discrimination. Canadian Journal of Physiology \& Pharmacology, 66, 439-454.

GiBson, J. J. (1962). Observations on active touch. Psychological Review, 69, 477-491.

Goodwin, A. W., John, K. T., \& Marceglia, A. H. (1991). Tactile discrimination of curvature by humans using only cutaneous information from the fingerpads. Experimental Brain Research, 86, 663-672.

GORDON, I. E., \& MORISON, V. (1982). The haptic perception of curvature. Perception \& Psychophysics, 31, 446-450.

Johnson, K. O., Hsiao, S. S., \& Twombly, I. A. (1996). Neural mechanisms of tactile form recognition. In M. S. Gazzaniga (Ed.), The cognitive neurosciences (pp. 253-267). Cambridge, MA: MIT Press.

KAPPERS, A. M. L., \& KoENDERINK, J. J. (1996). Haptic unilateral and bilateral discrimination of curved surfaces. Perception, 25, 739-749.

LaMotte, R. H., Srinivasan, M. A., Lu, C., \& Klusch-Petersen, A. (1994). Cutaneous neural codes for shape. Canadian Journal of Physiology \& Pharmacology, 72, 498-510.

Lederman, S. J., \& KlatzKy, R. L. (1987). Hand movements: A window into haptic object recognition. Cognitive Psychology, 19, $342-$ 368 .

LOOMIS, J. M., \& LedermaN, S. J. (1986). Tactual perception. In K. R. Boff, L. Kaufman, \& J. P. Thomas (Eds.), Handbook of perception and human performance: Vol. 2. Cognitive processes and performance (pp. 31.1-31.41). New York: Wiley.

Macmillan, N. A., \& Creelman, C. D. (1991). Detection theory: A user's guide. Cambridge: Cambridge University Press.

Matthews, P. B. C. (1988). Proprioceptors and their contribution to somatosensory mapping: Complex messages require complex processing. Canadian Journal of Physiology \& Pharmacology, 66, 430438.

PonT, S. C., Kappers, A. M. L., \& KoenderinK, J. J. (1995). Haptic discrimination of curved strips. In B. G. Bardy, R. J. Bootsma, \& Y. Guiard (Eds.), Studies in perception and action III (pp. 307-310). Hillsdale, NJ: Erlbaum.

Pont, S. C., Kappers, A. M. L., \& Koenderink, J. J. (1996). The influence of stimulus length on static haptic curvature discrimination. In A. M. L. Kappers, C. J. Overbeeke, G. J. F. Smets, \& P. J. Stappers (Eds.), Studies in ecological psychology (pp. 69-72). Delft: Delft University Press.

Pont, S. C., Kappers, A. M. L., \& Koenderink, J. J. (1997). Haptic curvature discrimination at several regions of the hand. Perception \& Psychophysics, 59, 1225-1240.

Press, W. H., Flannery, B. P., Teukolsky, S. A., \& Vetterling, W. T. (1988). Numerical recipes in C. Cambridge: Cambridge University Press.

TAN, H. Z., PANG, X. D., \& Durlach, N. I. (1992). Manual resolution of length, force, and compliance. Advances in Robotics, 42, 13-18.

VIRSU, V. (1971). Underestimation of curvature and task dependence in visual perception of form. Perception \& Psychophysics, 9 (3B), 339342 . 